International Journal of Bifurcation and Chaos, Vol. 12, No. 4 (2002) 783-813

(c) World Scientific Publishing Company

\title{
CLASSIFICATION OF BIFURCATIONS AND ROUTES TO CHAOS IN A VARIANT OF MURALI-LAKSHMANAN-CHUA CIRCUIT
}

\author{
K. THAMILMARAN and M. LAKSHMANAN \\ Centre for Nonlinear Dynamics, Department of Physics, \\ Bharathidasan University, Tiruchirappalli-620 024, India
}

Received May 14, 2001; Revised June 14, 2001

\begin{abstract}
We present a detailed investigation of the rich variety of bifurcations and chaos associated with a very simple nonlinear parallel nonautonomous LCR circuit with Chua's diode as its only nonlinear element as briefly reported recently [Thamilmaran et al., 2000]. It is proposed as a variant of the simplest nonlinear nonautonomous circuit introduced by Murali, Lakshmanan and Chua (MLC) [Murali et al., 1994]. In our study we have constructed two-parameter phase diagrams in the forcing amplitude-frequency plane, both numerically and experimentally. We point out that under the influence of a periodic excitation a rich variety of bifurcation phenomena, including the familiar period-doubling sequence, intermittent and quasiperiodic routes to chaos as well as period-adding sequences, occur. In addition, we have also observed that the periods of many windows satisfy the familiar Farey sequence. Further, reverse bifurcations, antimonotonicity, remerging chaotic band attractors, and so on, also occur in this system. Numerical simulation results using Poincaré section, Lyapunov exponents, bifurcation diagrams and phase trajectories are found to be in agreement with experimental observations. The chaotic dynamics of this circuit is observed experimentally and confirmed both by numerical and analytical studies as well PSPICE simulation results. The results are also compared with the dynamics of the original MLC circuit with reference to the two-parameter space to show the richness of the present circuit.
\end{abstract}

Keywords: Bifurcations; chaos; nonlinear electronic circuits.

\section{Introduction}

Nonlinear electronic circuits are turning out to be extremely convenient tools to understand the various phenomena underlying nonlinear dynamical systems through experimental, numerical and analytical studies [Chua et al., 1987; Lakshmanan \& Murali, 1996; Madan et al., 1993]. Chua's circuit is the most standing example in this regard, which has stimulated investigations along several directions on chaos, controlling, synchronization, and so on [Madan et al., 1993]. The most important characteristics of the Chua's circuit family is the piecewise-linear nature of the nonlinear element, which is a nonlinear resistor called Chua's diode. Very often such a piecewise-linear system can also model real physical devices like varactor diode, neon bulb, Josephson junction, etc. to yield quantitative results [Madan et al., 1993] and therefore the study of such systems is also of practical importance.

Most of the above type of circuits are autonomous and three-dimensional, represented by sets of three coupled first-order differential equations. However, it will be advantageous to consider even simpler nonlinear electronic circuits involving piecewise-linear elements and driven by periodic signals, as such systems are relatively easy 
to construct and to study both numerically and analytically. From this point of view Murali, Lakshmanan and Chua have introduced the simplest nonlinear nonautonomous circuit consisting of the familiar forced series LCR circuit to which the Chua's diode is connected in parallel [Murali et al., 1994]. The resultant dynamical equations are a set of two first-order coupled nonlinear differential equations with a forcing term included. The circuit exhibits several interesting dynamical phenomena including period-doubling bifurcations, chaos and periodic windows. However, in the parameter regimes investigated, it does not exhibit other important phenomena such as quasiperiodicity, intermittency, period-adding sequences, and so on. It will be quite valuable from the nonlinear dynamics point of view to construct a simple electronic circuit which exhibits a wide spectrum of dynamical phenomena. In this paper, we wish to point out that a rich variety of phenomena can be realized with a simple variant of the above MLC circuit, by connecting the Chua's diode to a forced parallel LCR circuit instead of the forced series LCR circuit. Just as the series and parallel LCR circuits are paradigmic second-order linear systems so also the MLC circuit and its variant may be considered as the second-order nonlinear systems which can capture a rich variety of dynamical phenomena. In particular, it turns out that the MLC variant circuit can exhibit not only the familiar period-doubling route to chaos and windows but also intermittent and quasiperiodic routes to chaos as well as period-adding sequences and Farey sequences, crisis, chaotic band mergings and so on.

Very recently, we have briefly discussed [Thamilmaran et al., 2000] the observation of a rich variety of bifurcations and chaos in the variant of the MLC circuit, for certain parametric choices. In the present paper we carry out a detailed experimental investigation of the circuit and obtain a two-parameter phase diagram, where the control parameters are the drive amplitude and frequency of the impressed periodic signal. The results are confirmed by means of an exhaustive numerical analysis supported by studies involving Poincaré surface of section, Lyapunov exponents, bifurcation diagrams and phase diagram. We also confirm some of our results by PSPICE simulation. In order to appreciate the richness of the dynamics of the present variant circuit, we compare its phase diagrams (both experimental and numerical) with the two-parameter phase diagrams of the original
MLC circuit (which was not obtained in the earlier studies). Finally we also point out that due to the piecewise-linear nature of the Chua's diode, the dynamical equations can be explicitly "integrated" in terms of elementary functions from which the numerical results can be further confirmed.

We have organized the paper as follows. Section 2 presents a brief introduction to the original MLC circuit and two-parameter phase diagrams are constructed corresponding to experimental and numerical results, so as to elucidate the dynamical behavior of this circuit. In Sec. 3, we introduce the experimental realization of a simple variant of the MLC circuit and demonstrate experimentally several bifurcations and chaos phenomena occurring in the system. We also present an experimental phase diagram in the drive amplitude-drive frequency parameter plane using observed data from the laboratory. Section 4 is devoted to the numerical simulation studies of the variant MLC circuit and the underlying chaotic dynamics and a corresponding phase diagram is obtained. A brief mention of the PSPICE simulation results is made in Sec. 5. Next, in Sec. 6, a study of fixed points, their linear stability and the explicit analytical solution of the dynamical equations of the variant MLC circuit are reported and the earlier results are confirmed. Finally, in Sec. 7, we summarize our results and indicate further directions.

\section{Dynamics of the MLC Circuit}

In order to appreciate the nature and importance of the variant MLC circuit, we first describe briefly the circuit realization of the original MLC circuit itself [Murali et al., 1994] and present twoparameter phase diagrams (not presented in the literature earlier) in order to bring out the dynamical properties of this circuit. We will also make use of this study to compare the dynamics of the proposed variant of the MLC circuit in the following sections.

\subsection{Realization of the $M L C$ circuit and experimental phase diagram}

The MLC circuit is the simplest nonlinear dissipative nonautonomous electronic circuit consisting of a forced series LCR circuit to which the Chua's diode, which is a nonlinear resistor, is connected 


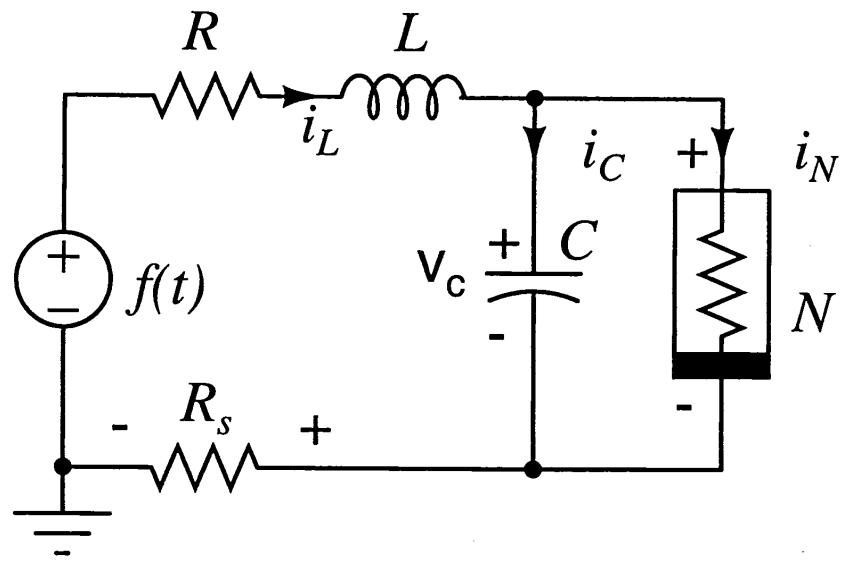

Fig. 1. Circuit realization of the simplest nonautonomous MLC circuit. Here $N$ is the nonlinear resistor (Chua's diode).

parallely. The circuit was originally introduced by Murali, Lakshmanan and Chua (MLC) [1994]. The circuit realization is given in Fig. 1. It is a classic configuration of a forced negative resistance oscilla- tor, where $N$ denotes a voltage-controlled nonlinear resistor described by the relation $i=g(v)$, which in this case is a Chua's diode [Cruz \& Chua, 1992]. Here $C$ is the capacitor, $R$ is a linear resistor, $L$ is an inductor while $R_{s}$ is a current sensing resistor and $f(t)(=f \sin \Omega t)$ is the external periodic forcing. Then the governing equations of this circuit for the voltage $v$ across the capacitor $C$ and the current $i_{L}$ through the inductor $L$ are given by the following set of two first-order coupled nonautonomous differential equations:

$$
\begin{aligned}
C \frac{d v}{d t} & =i_{L}-g(v), \\
L \frac{d i_{L}}{d t} & =-R i_{L}-R_{s} i_{L}-v+f \sin (\Omega t) .
\end{aligned}
$$

Here the characteristic function $g(v)$ is given by

$$
g(v)=G_{b} v+0.5\left(G_{a}-G_{b}\right)\left[\left|v+B_{p}\right|-\left|v-B_{p}\right|\right]
$$

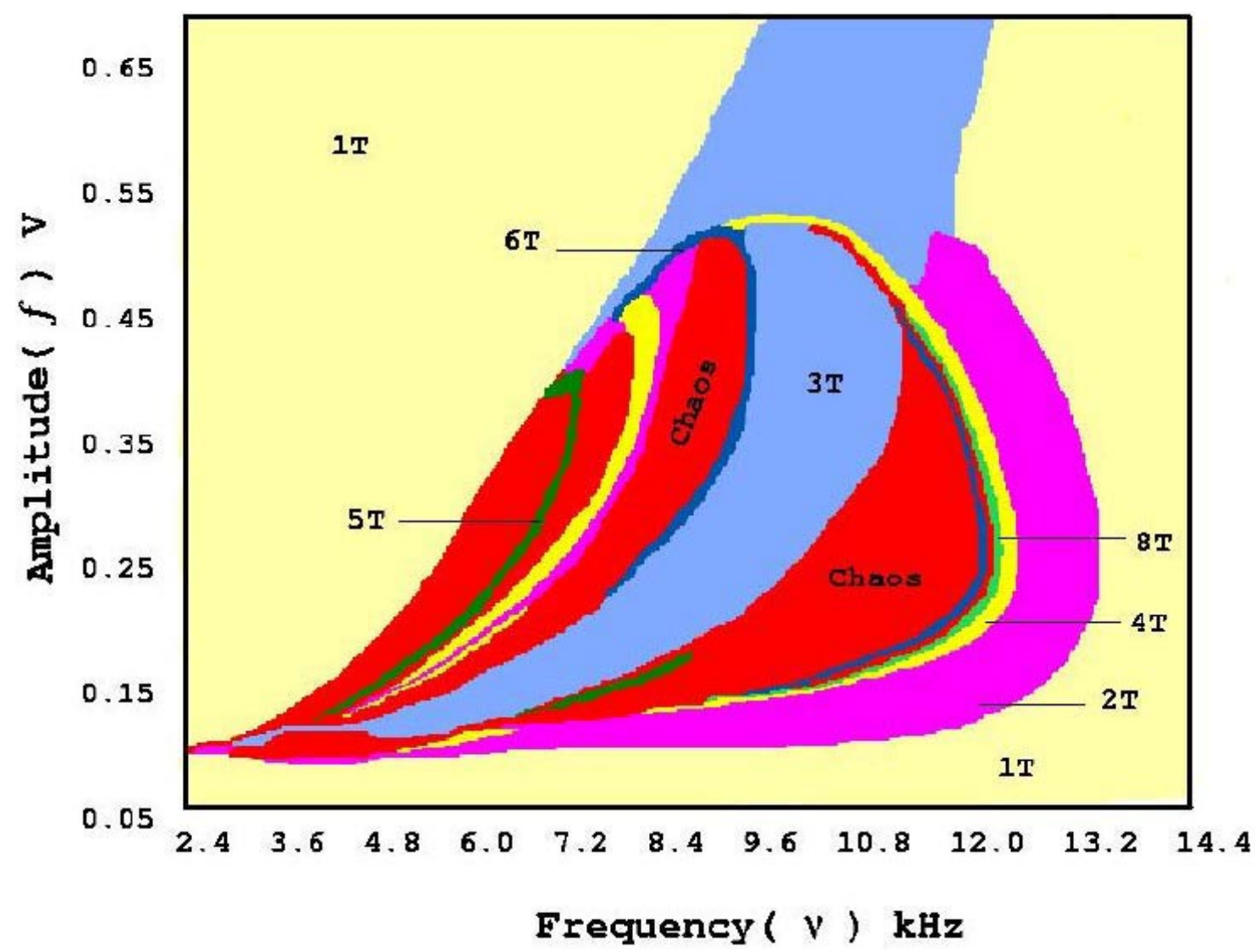

Fig. 2. Experimentally measured two-parameter bifurcation diagram of the dynamical behavior of the MLC circuit of Fig. 1 in the $(f-\nu)$ plane. Different attractors are color-coded as follows: light yellow, period-1; magneta, period-2; yellow, period-4; light green, period-8; blue, period-3 window; green, period-5 window; violet, period-6 window; red, chaos. 
The experimental design of the Chua's diode is well described by Kennedy [1992]. In Eqs. (1), $f$ is the amplitude and $\Omega$ is the angular frequency of the external periodic signal.
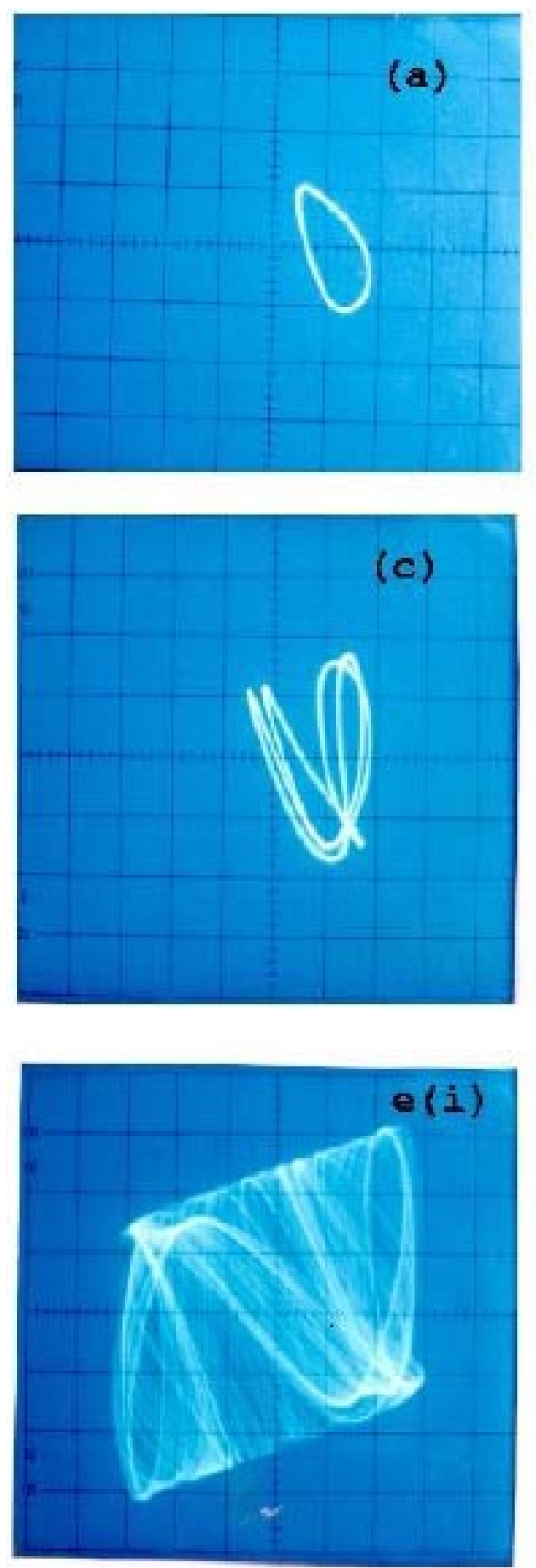

Now the behavior of the above circuit depends on five control parameters, namely the inductance $L$, the capacitance $C$, the linear resistance $R$, the amplitude $f$ and the frequency $\nu(=\Omega / 2 \pi)$ of the
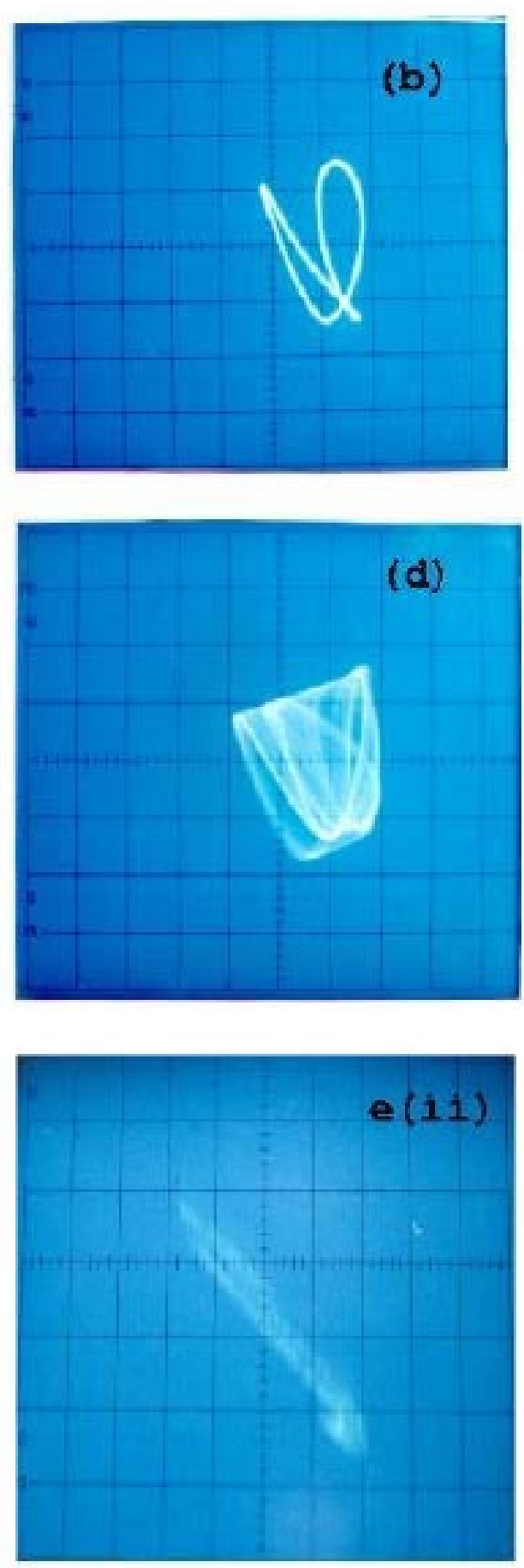

Fig. 3. Period-doubling scenario in the MLC circuit of Fig. 1: Phase portraits in the $(v-$ sin $\Omega t)$ plane, projected on the oscilloscope for fixed values of the circuit elements chosen as $C=10 \mathrm{nf}, L=18 \mathrm{mH}, R=1340 \Omega, R_{s}=20 \Omega$ and frequency $\nu=8.8 \mathrm{kHz}$ for increasing amplitude, $f$ : (a) period- $1 ; f=0.052 \mathrm{~V}$, (b) period-2; $f=0.087 \mathrm{~V}$, (c) period-4; $f=0.092 \mathrm{~V}$, (d) one-band chaos; $f=0.105 \mathrm{~V}$, (ei) double-band chaos; $f=0.138 \mathrm{~V}$, (eii) the strobed Poincaré map in the $\left(v-i_{L}\right)$ plane of (i), (f) period-3 window; $f=0.25 \mathrm{~V}$ and (g) period-1 boundary; $f=0.55 \mathrm{~V}$. 

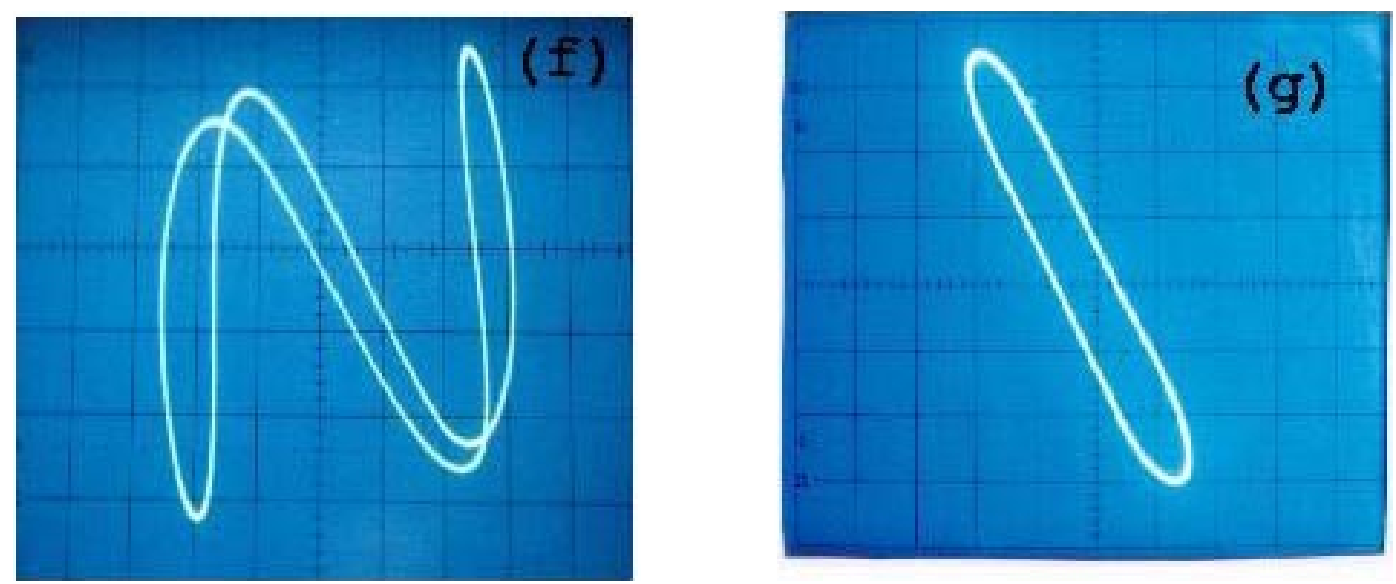

Fig. 3. (Continued)
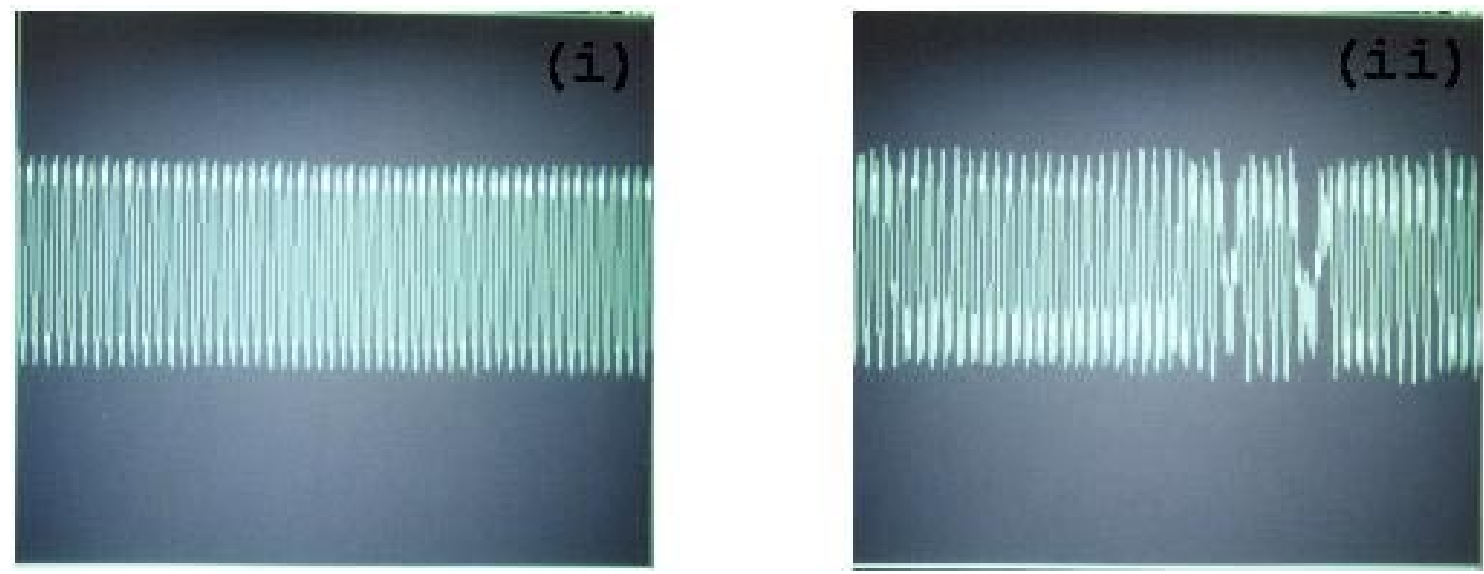

Fig. 4. (i) Time series plot for a stable period-3 window for $f=0.117 \mathrm{~V}$ and $\nu=5.689 \mathrm{kHz}$ and (ii) the waveform $v(t)$ for type I intermittency near the period-3 window for $f=0.117 \mathrm{~V}$ and $\nu=5.697 \mathrm{kHz}$.

external periodic signal, besides the parameters associated with Chua's diode (which we usually fix at definite values). The values of the linear circuit elements are fixed as $R=1340 \Omega, R_{s}=20 \Omega$, $L=18 \mathrm{mH}, C=10 \mathrm{nf}$ and the parameters of the Chua's diode are chosen as $G_{a}=-0.76 \mathrm{mS}$, $G_{b}=-0.41 \mathrm{mS}$ and $B_{p}=1.0 \mathrm{~V}$. In their original work, Murali et al. [1994] have studied the dynamics of the circuit given by Eq. (1) by fixing the frequency, $\nu(=\Omega / 2 \pi)$, at $8.89 \mathrm{kHz}$ and varying the amplitude $f$ in the range $(0.05 \mathrm{~V}, 0.7 \mathrm{~V})$. In our present experimental study, both the amplitude $f$ and the frequency $\nu(=\Omega / 2 \pi)$ of the forcing source are used as the control parameters. By scanning both $f$ and $\nu$, one can identify various attractors, starting from the d.c. equilibrium, then Hopf bifurcation to a limit cycle, and then period-doubling sequences to a spiral type chaotic attractor, a double-scroll type attractor, periodicwindows, and so on. In addition, a few other interesting dynamical phenomena can also be identified by a careful study through combined amplitude and frequency scanning procedures.

Based on our detailed experimental observations of the voltage $v$ across the capacitor $C$ and the current $i_{L}$ through the inductor $L$ and the associated transitions as the amplitude $(f)$ and frequency $(\nu)$ are varied, a profile of the attractors in the form of a two-parameter bifurcation diagram in the $f-\nu$ (drive amplitude-drive frequency) parameter plane has been constructed as shown in Fig. 2.

The chosen range for our experimental study is limited to $0.05 \mathrm{~V} \leq f \leq 0.7 \mathrm{~V}$ and $2.4 \mathrm{kHz} \leq$ 
$\nu \leq 14.4 \mathrm{kHz}$. In Fig. 2 each colored region denotes a particular type of steady state behavior: for example, light yellow, period-1 attractor; magneta, period-2 attractor; yellow, period-4 attractor; light green, period-8 attractor; blue, period-3 window; green, period-5 window; violet, period-6 window; and red, chaotic attractor. Particularly, the standard period-doubling bifurcation sequence, intermittency route to chaos and chaos-periodic windowchaos type of transitions have been observed in different regions of the drive amplitude and frequency. For example, typical period-doubling bifurcation sequence occurs in the frequency range $\nu=(8.30 \mathrm{kHz}, 8.95 \mathrm{kHz})$ and amplitude range $f=(0.05 \mathrm{~V}, 0.15 \mathrm{~V})$. More specifically, by increasing the amplitude $f$ from zero upwards, at fixed frequency $8.8 \mathrm{kHz}$, the circuit in Fig. 1 is found to exhibit a sequence of bifurcations. Starting from d.c. equilibrium, the solution bifurcates through a Hopf bifurcation to a limit cycle, and then by period-doubling sequence to a spiral Chua's attractor, a double-scroll Chua's chaotic attractor, periodic-windows, boundary crisis, etc., as illus- trated in Fig. 3 in terms of $(v-\sin \Omega t)$ plots and the corresponding Poincaré map in the $\left(v-i_{L}\right)$ plane for double band chaotic attractor as observed in the laboratory.

Further, the occurrence of type I intermittency near a period-3 window is shown in Fig. 4. A portion of the time dependence of the waveform $v$ for a period-3 window for the parameters $f=0.117 \mathrm{~V}$ and $\nu=5.689 \mathrm{kHz}$ is shown in Fig. 4(i). With further increase of frequency to $\nu=5.697 \mathrm{kHz}$, one finds that the periodic oscillations are interrupted by intermittent voltage bursts as shown in Fig. 4(ii), giving rise to type I intermittency route to chaos.

Finally, a series of appearance and disappearance of chaos followed by periodic-windows exists in the range $\nu=(4.7 \mathrm{kHz}, 8.9 \mathrm{kHz})$ and $f=(0.15 \mathrm{~V}$, $0.32 \mathrm{~V})$.

\subsection{Computer confirmation}

Rescaling Eq. (1) as $v=x B_{p}, i_{L}=y G B_{p}, G=1 / R$, $\omega=(\Omega C / G)$ and $t=\tau C / G$ and then redefining $\tau$ as $t$, the following set of normalized equations

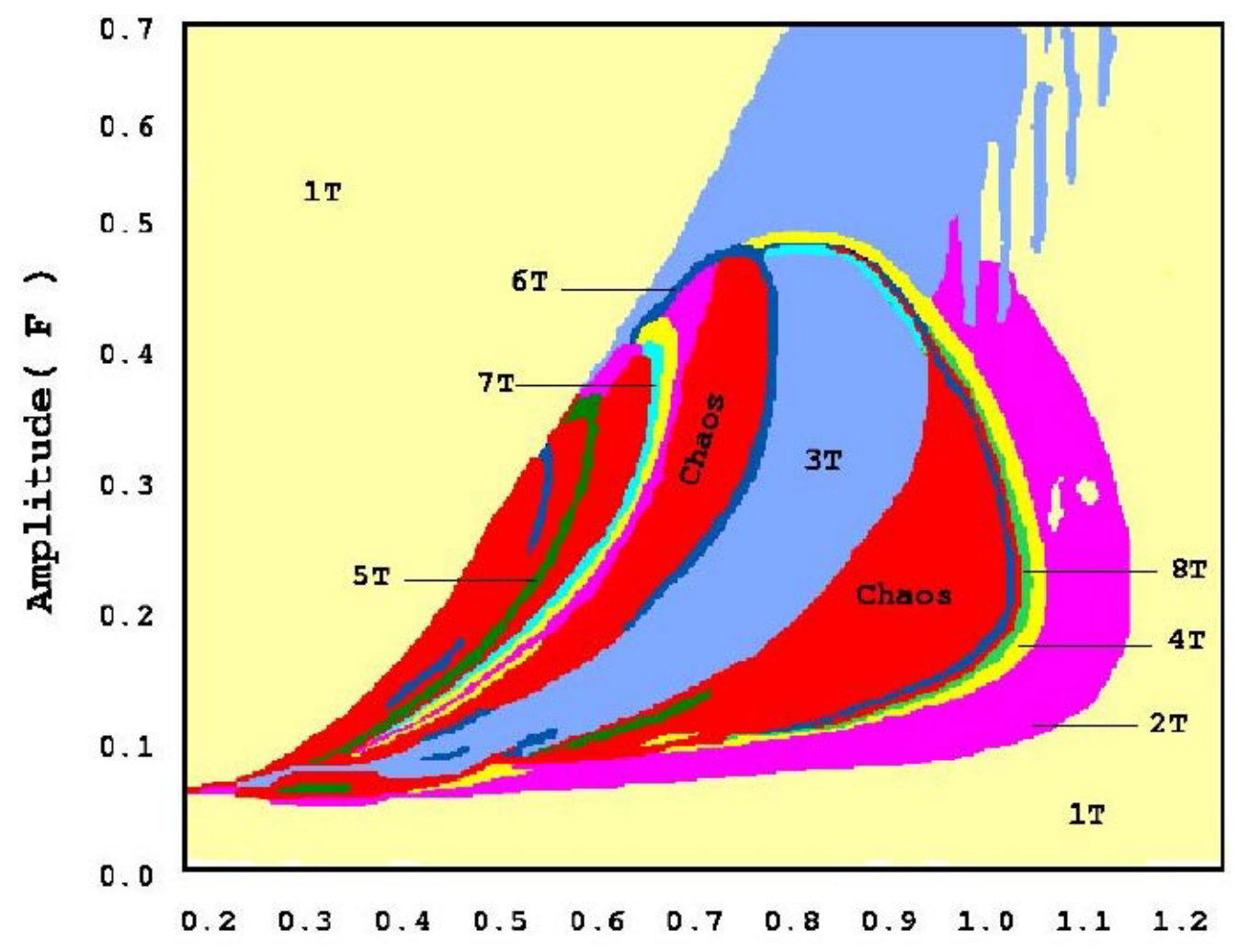

\section{Frequency $(\omega)$}

Fig. 5. Computer generated phase diagram in the $(F-\omega)$ plane for the MLC circuit, Eqs. (2). Each colored region denotes a particular type of steady-state behavior as in Fig. 2 . 

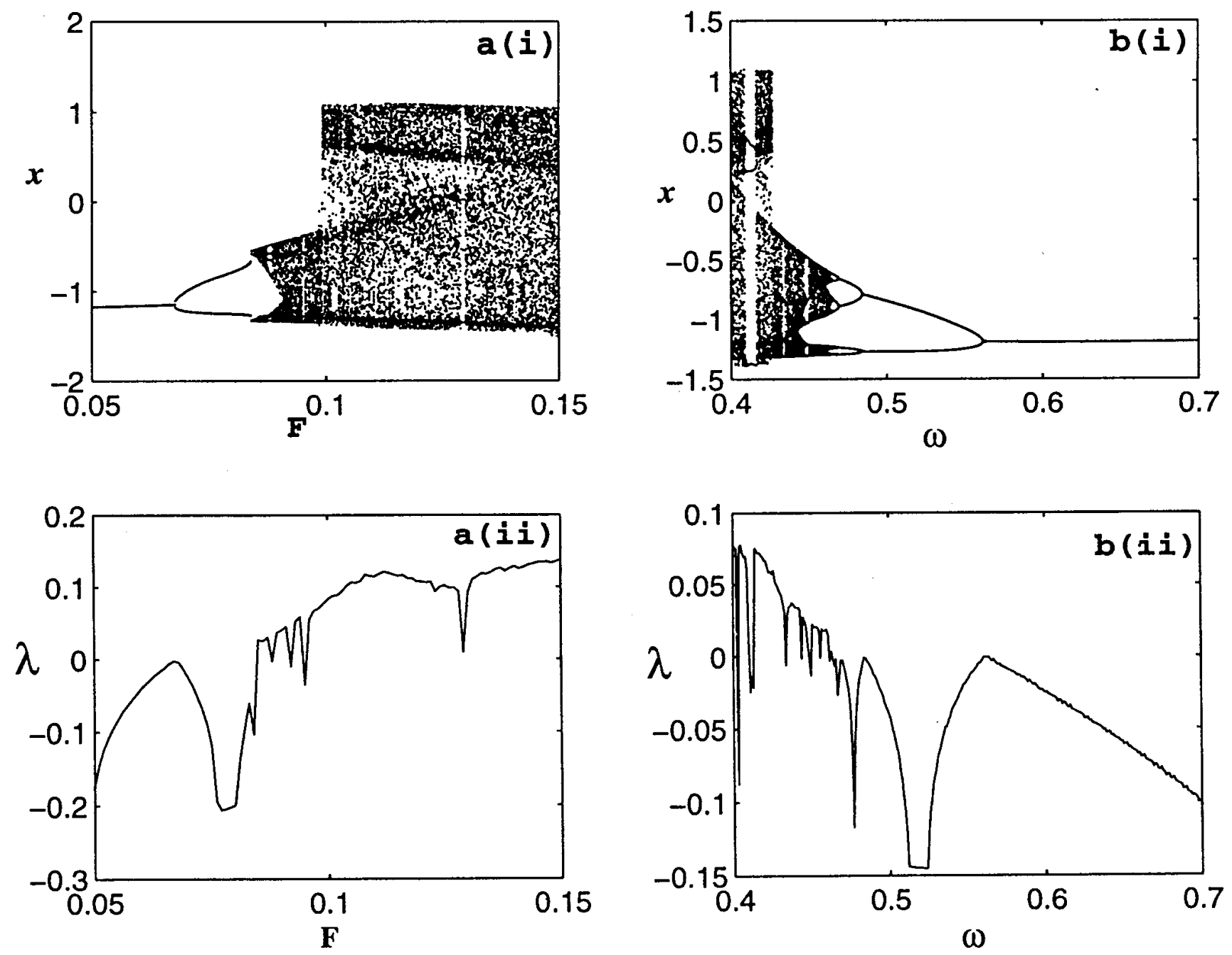

Fig. 6. MLC circuit (numerical) (ai) Amplitude scanning: one-parameter bifurcation diagram in the $(F-x)$ plane and (ii) maximal Lyapunov exponent in the $\left(F-\lambda_{\max }\right)$ plane. The value of the frequency has been fixed at $\omega=0.72$. (bi) Frequency scanning: one-parameter bifurcation diagram in the $(\omega-x)$ plane and (ii) maximal Lyapunov exponent in the $\left(\omega-\lambda_{\max }\right)$ plane. The value of the amplitude has been fixed at $F=0.063$.

are obtained,

$$
\begin{aligned}
& \dot{x}=y-g(x), \\
& \dot{y}=-\beta y-\nu \beta y-\beta x+F \sin (\omega t), \quad\left(\cdot=\frac{d}{d t}\right)
\end{aligned}
$$

where

$$
g(x)=b x+0.5(a-b)(|x+1|-|x-1|)
$$

and $\beta=\left(C / L G^{2}\right), \nu=G R_{s}, F=f \beta / B_{p}, a=$ $G_{a} / G$, and $b=G_{b} / G$.

The dynamics of (2) now depends on the parameters $\beta, \nu, a, b, \omega$ and $F$. In the original simulations, Murali et al. [1994] have used the rescaled circuit parameters corresponding to the previous experimental parameters at the values $\beta=1.0$, $\nu=0.015, a=-1.02$ and $b=-0.55$ and analyzed numerically the dynamics for increasing values of the driving amplitude $F$ for fixed $\omega(=0.75)$ only. In this case the system has been found to exhibit the familiar period-doubling bifurcation route to chaos, followed by periodic windows, etc. as already observed from the experimental circuit. In order to compare the dynamics of the MLC circuit with that of the variant circuit, we have now worked out a two-parameter phase diagram (Fig. 5) in the $(F, \omega)$ plane by numerically integrating Eq. (2) using a fourth-order Runge-Kutta algorithm with parameters $\beta, \nu, a$ and $b$ fixed as above, while varying the drive amplitude $F$ in the range $0.0 \leq F$ 

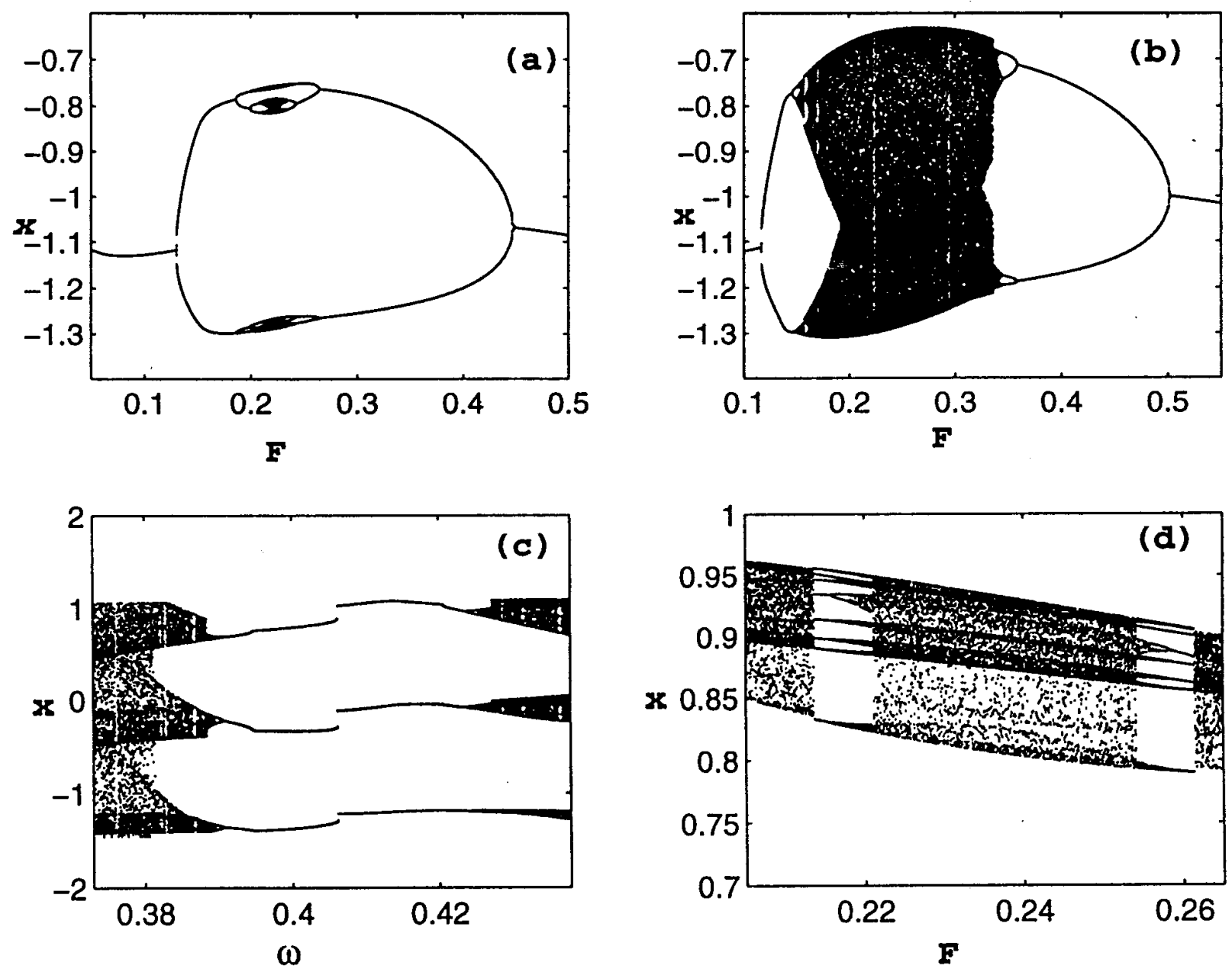

Fig. 7. Antimonotonicity in the MLC circuit: (a) One parameter bifurcation diagram in the $(F-x)$ plane showing an infinite period bubble and chaotic structure at fixed frequency $\omega=1.01$, through forward and reverse period-doubling bifurcations. (b) Bifurcation diagram in the $(F-x)$ plane for band merging at $\omega=0.975$. The merging point is $F=0.195$. (c) Bifurcation diagram in the range $0.37 \leq \omega \leq 0.44$ of the parameter $\omega$ for a reverse period-3 bubble at fixed $F=0.075$. (d) Bifurcation diagram in the $(F-x)$ plane showing a period-8 bubble for $0.205 \leq F \leq 0.265$ at fixed $\omega=0.998$.

$\leq 0.7$ and frequency $\omega$ in the range $0.2 \leq \omega \leq$ 1.2. In this diagram, again each colored region denotes a particular type of steady-state behavior as in the case of Fig. 2. The transitions are characterized by tracing the time evolutions, phase portrait, Poincaré map, and Lyapunov exponents [Murali et al., 1994]. Various dynamical phenomena are traced out by two different scanning procedures: (i) varying $\omega$ at a fixed $F$ (frequency scanning) and (ii) varying $F$ at a fixed $\omega$ (amplitude scanning). We also note that the phase diagram (Fig. 5) constructed from the present numerical analysis closely resembles the one constructed from experimental studies (Fig. 2), though Fig. 5 has much more finer details due to obvious reasons.

To start with, in Fig. 5, we observe that for $F=0$ and $\omega=0$ the system asymptotically approaches a stable fixed point (see Sec. 6 below). Then for low $F$ values and all $\omega$ values in the range of study, a stable period $T(=2 \pi / \omega)$ limit cycle occurs, which persists upto $F=0.047$ and $\omega=1.2$ (see Fig. 5). By increasing both $F$ and $\omega$, we have observed a series of appearance and disappearance of periodic (phase-locking) and chaotic attractors in the amplitude and frequency scanning. In particular, the following phenomena are found:

(i) The standard period-doubling bifurcation sequences and chaotic attractors have been observed in the regions of lower drive amplitude $(F)$ and certain regions of low and middle frequency $(\omega)$ values. For example, at fixed frequency $\omega=0.72$ on increasing the amplitude $F$ in the range $F=$ $(0.05,0.15)$ along the upward direction, chaos via 

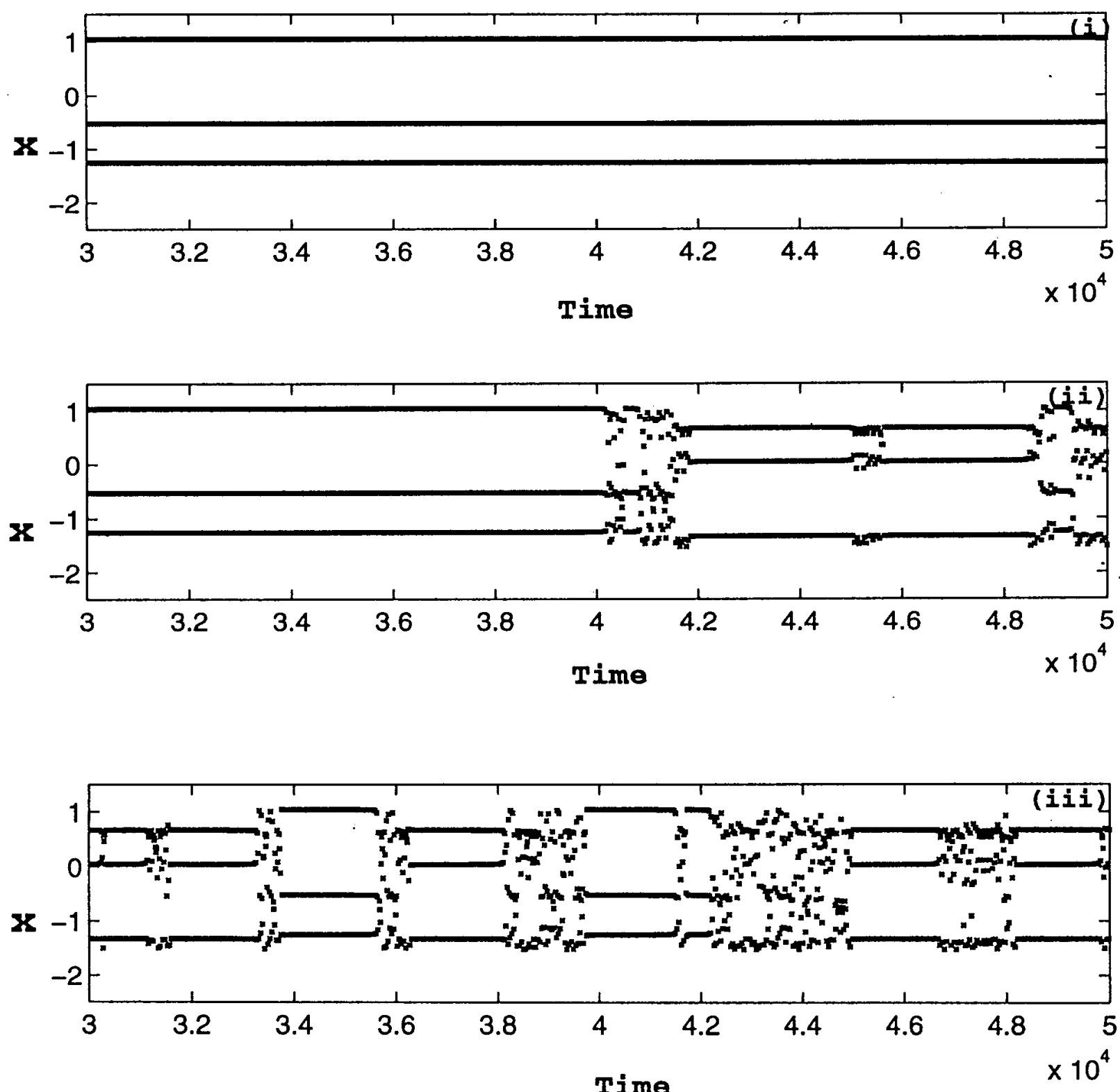

Fig. 8. Type I intermittency in the MLC circuit: Poincaré plot for $F=0.117$ and (i) $\omega=0.48675$ for a stable period-3 window, (ii) $\omega=0.486748$ and (iii) $\omega=0.486746$ for intermittent behavior.

period-doubling bifurcations occurs - see the phase diagram (Fig. 5) and the one-parameter bifurcation diagram [Fig. 6(ai)] in the $F-x$ plane, which clearly indicate the familiar period-doubling bifurcation sequence, chaos, windows, etc. In Fig. 6(aii) the corresponding maximal Lyapunov exponent is plotted as a function of $F$. Similarly, as an example, by making an $\omega$-scanning for fixed $F=0.063$, in the range $\omega=(0.4,0.7)$, one can observe the period-doubling route to chaos in the reverse sense [Fig. 6b)]. (ii) Next, we come across the phenomenon of antimonotonicity (see e.g. [Lakshmanan \& Murali, 1996]), wherein the periodic orbits can be created as well destroyed via reverse bifurcation sequences in different ways (see also Sec. 4.3.5 below). They are as follows.

(a) Moving upward along $\omega=1.01$ in Fig. 5, one can identify a sequence of periodic orbits $1,2,4$, 8 , etc., then a chaotic region with considerable fine structure, followed by reverse period-doubled orbits $8,4,2,1$ in the region $F=(0.07,0.5)$ as shown in 

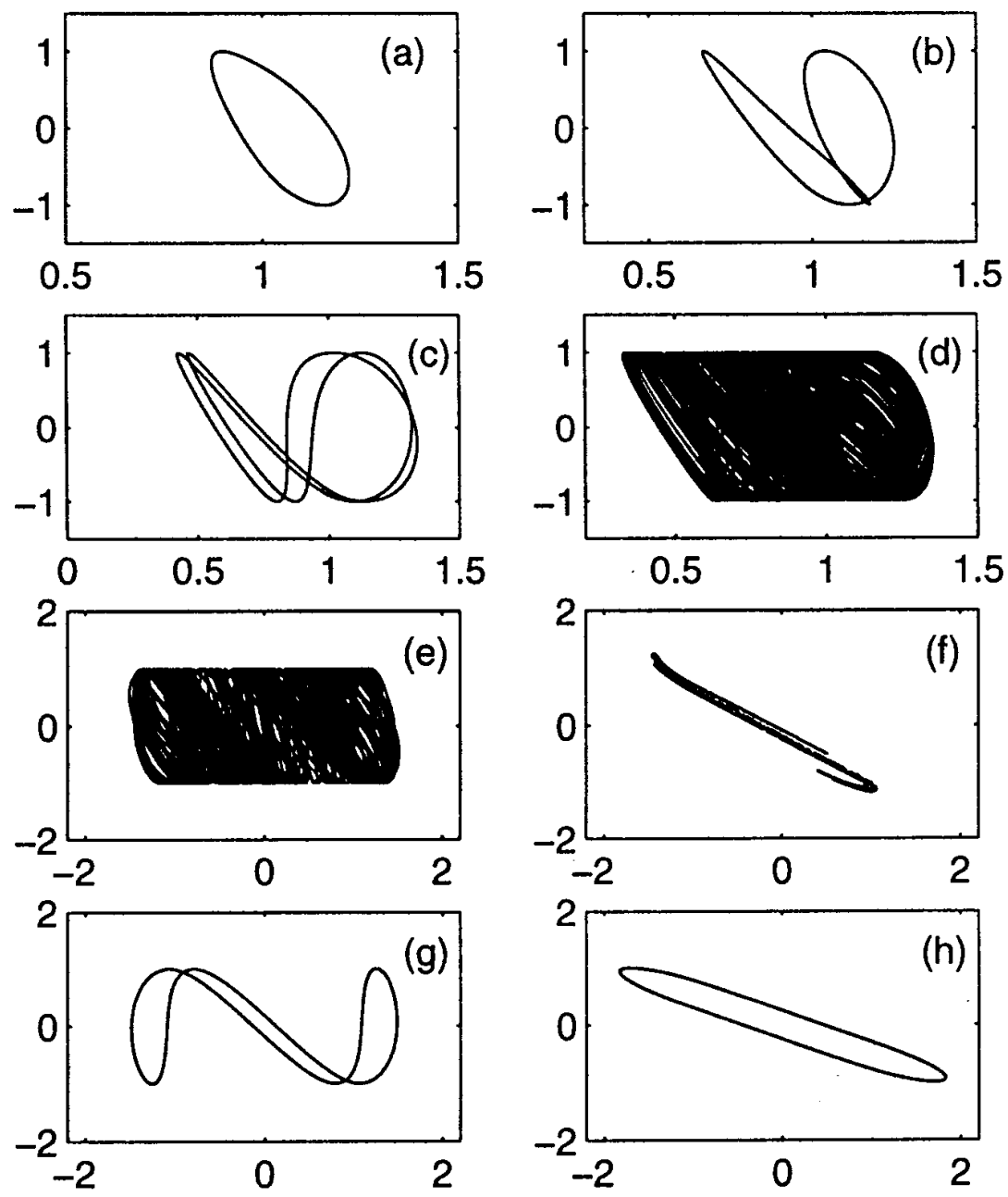

Fig. 9. MLC Circuit (numerical analysis): Phase portraits in the $(x-\sin \omega t)$ plane of Eq. (2) for the parameters $\beta=1.0$, $\nu=0.015, a=-1.02, b=-0.55$ and $\omega=0.72$. (a) period- $1 ; F=0.059$, (b) period- $2 ; F=0.075,(\mathrm{c})$ period-4; $F=0.082$, (d) one-band chaos; $F=0.098$, (e) double-band chaos; $F=0.128$, (f) Poincaré map in the $(x-y)$ plane of (e), (g) period-3 window; $F=0.28$, and (h) period-1 boundary; $F=0.56$.

Fig. 7(a), having an infinite period bubble structure. Considering a higher value of $\omega$, during $F$ scanning $[F=(0.07,0.5)]$, one comes across various finite period- $m$ bubbles. For example at $\omega=1.025$, a period- 8 bubble, at $\omega=1.05$, a period- 4 bubble and at $\omega=1.1$, a primary period-2 bubble occur.

(b) It is known that two or more chaotic band attractors of a system with symmetries can merge to form a single chaotic band attractor as the control parameter $\omega$ is varied through a critical value. This phenomenon is called band merging crisis [Ott, 1993]. In this case, the new chaotic band attractor can be larger in size than the union of the chaotic attractors before the period-doubling. Such band merging attractors also exist in the present case as well. For example, at fixed frequency $\omega=0.975$, as the amplitude $F$ is increased in the range $F=$
$(0.1,0.55)$, we observe merging of chaotic regions, between the forward and reverse period-doubling sequences [Fig. $7(\mathrm{~b})]$.

(c) In addition, we come across reverse period- $m$ bubbles also in the present system, both during $\omega$ scanning and $F$ scanning. For example, as $\omega$ is increased in the range $\omega=(0.37,0.44)$, at the fixed amplitude $F=0.075$, chaotic behavior is followed by a complete sequence of reverse period-doubling cascades of period-3 oribit; then a complete perioddoubling sequence of period-3 orbit is followed by chaotic oscillations. The pattern may be called a reverse period-3 bubble - see Fig. 7(c). In additon, period- $m$ bubbles also occur between two chaotic regions in this circuit. For example, as $F$ is increased in the range $F=(0.205,0.265)$, at the fixed frequency $\omega=0.998$, a tangent bifurcation of 
periodic orbit with period-8 is followed by a complete sequence of period-doubling bifurcations; then, after an interval with chaotic behavior, there is a reverse period-doubling sequence, ending in a periodic orbit with period-8 which then again undergoes a tangent bifurcation to chaos. This pattern obviously corresponds to a period- 8 bubble — see Fig. 7(d).

(iii) Finally, we have also confirmed the existence of type I intermittency route as in the case of experimental observations. At fixed amplitude, $F=0.117$, and with a decrease of frequency in the range $\omega=(0.48675,0.486746)$, one observes type I intermittency as shown in Fig. 8. At $\omega=0.48675$, the system has a period-3T attractor. The corresponding Poincare points in the state $x$ are shown in Fig. 8(i). As $\omega$ is gradually decreased, finite intervals of regular oscillations are interupted by intermittent bursts of irregular oscillations Fig. 8(ii). The bursts are more prominent in Fig. 8(iii), with the duration of regular oscillations decreasing in size and the bursts becoming more frequent. Finally, at $\omega=0.48674$ a fully chaotic state is attained. The average laminar length $(\langle l\rangle)$ of this type of intermittency is found to comply with the law $(\langle l\rangle) \sim \varepsilon^{-\alpha}$, with $\alpha=0.473$, where $\varepsilon=\left(\omega_{c}-\omega\right)$ and $\omega_{c}$ is the bifurcation threshold.

Finally, in order to compare the experimental results with the numerical analysis explicitly, we present in Fig. 9 the counterparts of the attractors obtained experimentally in Fig. 3. In the $(x-\sin \omega t)$ plane, these are obtained numerically by increasing the frequency $(F)$ from zero upwards at the fixed frequency $\omega=0.72$.

\section{A Variant of the MLC Circuit and Its Dynamics: Experimental Study}

The simplest dissipative nonautonomous nonlinear circuit discussed in the previous section admits a number of interesting bifurcations and chaotic behavior in the parameter regions considered. However, the phase diagram does not contain other interesting attractors such as the quasiperiodic (torus) attractor and bifurcation routes like periodadding sequences, Farey sequences, torus breakdown, etc. at least for the parameteric choices we have made and in the region of the phase diagrams considered. It will be valuable to identify and to construct a very simple nonlinear electronic circuit which admits as many interesting attractors and bifurcations as possible for chosen parameteric values and in the region of interest in the control parameters. We now present such a circuit which is nothing but a simple variant of the MLC circuit presented earlier.

\subsection{Circuit realization of the variant of $M L C$ circuit}

The present set up consists of a forced parallel LCR circuit, instead of the series LCR circuit considered in the earlier section, to which the Chua's diode is again connected parallely. The circuit realization of the proposed simple nonautonomous circuit is shown in Fig. 10. It again consists ${ }^{1}$ of a capacitor $(C)$, an inductor $(L)$, a resistor $(R)$, an external periodic-forcing voltage source and only one nonlinear element $(N)$, namely, the Chua's diode (discussed in the previous section). The resulting circuit can be considered as another important and very simple dissipative second-order nonautonomous nonlinear circuit. By applying the Kirchhoff's laws to this circuit (Fig. 10), the governing equations for the voltage $v$ across the capacitor $C$ and the current $i_{L}$ through the inductor $L$ are represented by the following set of two first-order coupled nonautonomous differential equations,

$$
\begin{aligned}
C \frac{d v}{d t} & =\frac{1}{R}(f \sin (\Omega t)-v)-i_{L}-g(v), \\
L \frac{d i_{L}}{d t} & =v
\end{aligned}
$$

where $g(\cdot)$ is the piecewise-linear function given by Eq. (1c) representing the Chua's diode as discussed under Eq. (1). In Eq. (3), as before, $f$ is the amplitude and $\Omega$ is the angular frequency of the external periodic signal.

We have performed our experimental study by fixing the three parameters $L, C$ and $R$ as in Fig. 10 at $L=445 \mathrm{mH}, C=10.15 \mathrm{nf}, R=1475 \Omega$ and varying the driving amplitude $(f)$ and frequency $(\nu)=\Omega / 2 \pi$ of the external periodic signal which are used as the control parameters. The parameters of

\footnotetext{
${ }^{1}$ Note that we have not included a current sensing resistor, $R_{s}$, here as all our studies in the following is made in the $(v-\sin \Omega t)$ plane; if needed, it can always be included.
} 


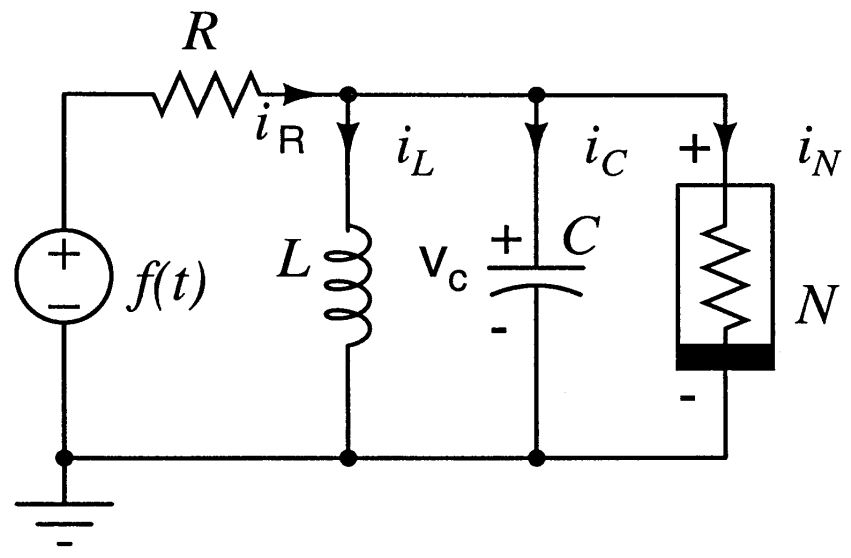

Fig. 10. Circuit realization of the variant of the MLC circuit (N: Chua's diode).

the Chua's diode are also fixed here at the same values as in the case of Eq. (1). We vary the amplitude $f$ in the range $(0.15 \mathrm{~V} \leq f \leq 0.5 \mathrm{~V})$ and the frequency $(\nu)$ in the range $(0.95 \mathrm{kHz} \leq \nu \leq$ $1.65 \mathrm{kHz})$.
We begin our experimental study of the circuit in Fig. 10 by choosing the driving amplitude at $f=0$ and frequency $\nu=0$ (corresponding to the autonomous case), where now a limit cycle attractor is observed, in contrast to the case of Eq. (1), where only a stable fixed point exists for the chosen parametric values. By increasing the control parameters from zero upwards, the circuit behavior of Fig. 10 is found to transit from a limit cycle attractor to a quasiperiodic (torus) attractor which then transits to chaos via torus breakdown, followed by periodic windows, period-adding, Farey and period-doubling sequences, boundary crisis, etc. These results are summarized in the constructed phase diagram (Fig. 11) in the $(f-\nu)$ plane.

\subsection{Bifurcation diagram in the $(f, \nu)$ plane}

In Fig. 11, again each colored region denotes a particular type of steady-state behavior as in the case

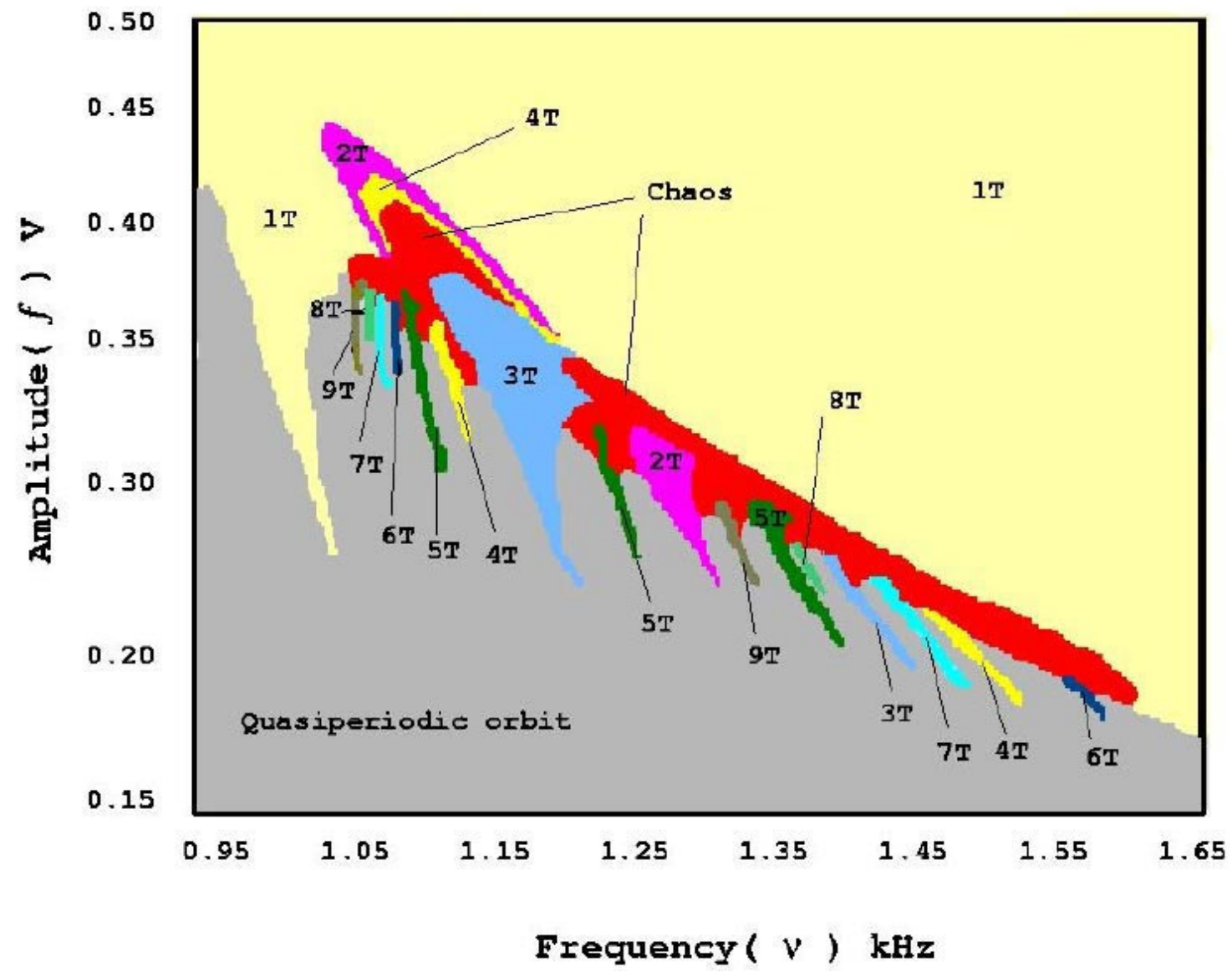

Fig. 11. Experimental phase diagram of the dynamical behavior of the circuit of Fig. 10 in the $(f-\nu)$ plane. Each colored region denotes a particular type of steady-state behavior as in Figs. 2 and 5. Additionally, gray color denotes quasiperiodic attractor. Numbers indicate the periods of windows. 
of Figs. 2 and 5. Additionally, gray color corresponds to quasiperiodic orbits and some other periodic windows are indicated by the corresponding periods. Specifically from our experimental studies we have observed the following properties: (a) quasiperiodic (torus) route and torus breakdown to chaos (b) period-adding scenario (c) perioddoubling bifurcations and (d) intermittency route to chaos, when both the driving amplitude $(f)$ and frequency $(\nu)$ of external periodic signal are varied, as discussed below.

\subsubsection{Quasiperiodic and torus breakdown to chaos}

Quasiperiodic attractors have been observed in several nonlinear dynamical systems and circuits [Kaneko, 1986; Matsumoto et al., 1987; Thompson \& Stewart, 1988; Inaba \& Mori, 1991; Murali \& Lakshmanan, 1992]. Generally, if the ratio of the two frequencies (driving frequency and circuit signal frequency) are irrational then the circuit can exhibit quasiperiodic motion. Such a behavior has been observed in the present variant MLC circuit (Fig. 10) for all values of $\nu$ in the range of study (Fig. 11), while confining the driving amplitude $(f)$ to the range $f=(0.15 \mathrm{~V}, 0.225 \mathrm{~V})$. Figure $12(\mathrm{a})$ shows the time series plot of the limit cycle for $f=0$, while Fig. 12(bi) shows a typical quasiperiodic attractor for $f=0.22 \mathrm{~V}$ and $\nu=1.116 \mathrm{kHz}$ in the $(v-\sin \Omega t)$ plane whose orbit in the associated Poincaré map is a closed curve [see Fig. 12(bii)]. Beyond the above mentioned region, we have observed a series of appearance and disappearance of the quasiperiodic (torus) and periodic (phase-locked) attractors alternately while varying both the amplitude $f$ and frequency $\nu$ of the external periodic signal, as seen in the phase diagram, Fig. 11. Except for the diagonal island structure with dentritic fine structures, the parametric space is occupied by quasiperiodic orbits below the island and period $1 \mathrm{~T}$ orbit above the island. The island and the dentrites themselves are associated with very many bifurcation phenomena, including period-doubling, period-adding and Farey sequences, and chaos, as described below.

Besides, in our experiments, we have also observed the breaking up of the quasiperiodic motion leading to chaos, followed by periodic windows both during driving amplitude $(f)$ scanning and frequency $(\nu)$ scanning. For example, we have observed this behavior at fixed $f=0.385 \mathrm{~V}$ on in- creasing the frequency, $\nu$, in the range $(1.025 \mathrm{kHz}$, $1.09 \mathrm{kHz}$ ) along the horizontal direction. Similarly, at fixed $\nu=1.36 \mathrm{kHz}$ an increase in the amplitude in the range $(0.26 \mathrm{~V}, 0.33 \mathrm{~V})$ along the upward direction leads to a torus breakdown and then to chaos for $f \geq 0.268 \mathrm{~V}$, finally ending up in period-1 orbit.

\subsubsection{Period-adding and Farey sequences}

Next, we observe the phenomenon of period-adding sequences as in the case of some negative resistance oscillators [Chua et al., 1986; Kaneko, 1986; Kennedy et al., 1989]. As noted above, at the lower end of the island structure in Fig. 11, windows of consecutive periods are separated by quasiperiodic attractors such that for a fixed $f$ we obtain a stable period- $n$ attractor $(n=1,2,3, \ldots)$ followed by a quasiperiodic attractor, and then a stable period- $(n+1)$ orbit and so on. During experiments with the present circuit, we observed many periodadding sequences of periodic windows of consecutively increasing periods. In particular, a periodadding sequence exists for higher drive amplitudes, $f=(0.35 \mathrm{~V}, 0.375 \mathrm{~V})$, and lower frequency values, $\nu=(1.025 \mathrm{kHz}, 1.15 \mathrm{kHz})$. If we look at Fig. 11, a succession of periodic windows, whose periods increase exactly by one, appears when the external frequency is decreased along the horizontal direction at fixed amplitude. For example, at fixed $f=(0.37 \mathrm{~V})$ we have observed the periodic windows of all orders starting from period-3 to period-9 as the frequency is decreased in the range $\nu=(1.03 \mathrm{kHz}, 1.1 \mathrm{kHz})$ along the horizontal direction. The transition from one periodic window to another is initiated by a quasiperiodic oscillation followed by a recovery to the next periodic state and so on as shown in Fig. 11.

In addition, we have found that the periods of some of the windows at the lower half of the island satisfy the Farey sequence [Kaneko, 1986; Murali \& Lakshmanan, 1991] in the quasiperiodic regions. For instance, we find that in the quasiperiodic region in Fig. 11, when $f=(0.28 \mathrm{~V}, 0.3 \mathrm{~V})$ and $\nu=(1.15 \mathrm{kHz}, 1.26 \mathrm{kHz})$, between $3 T$ and $2 T$ windows there is a $5 T(=3+2)$ window; and in the region $f=(0.25 \mathrm{~V}, 0.27 \mathrm{~V}), \nu=(1.35 \mathrm{kHz}$, $1.41 \mathrm{kHz})$, between $5 T$ and $3 T$ windows there is a $8 T(=5+3)$ window. One can observe a few other such sequences with some effort in the dentritic region. 


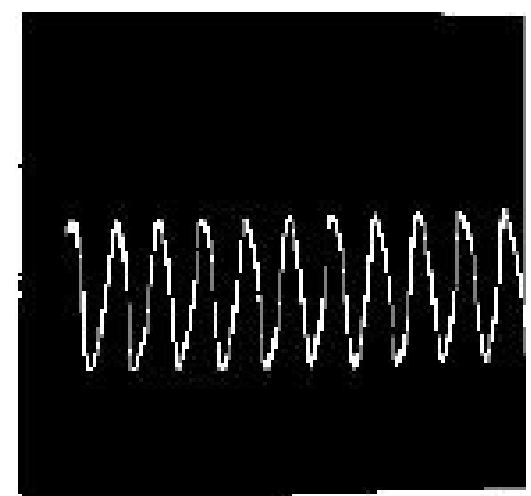

(a)
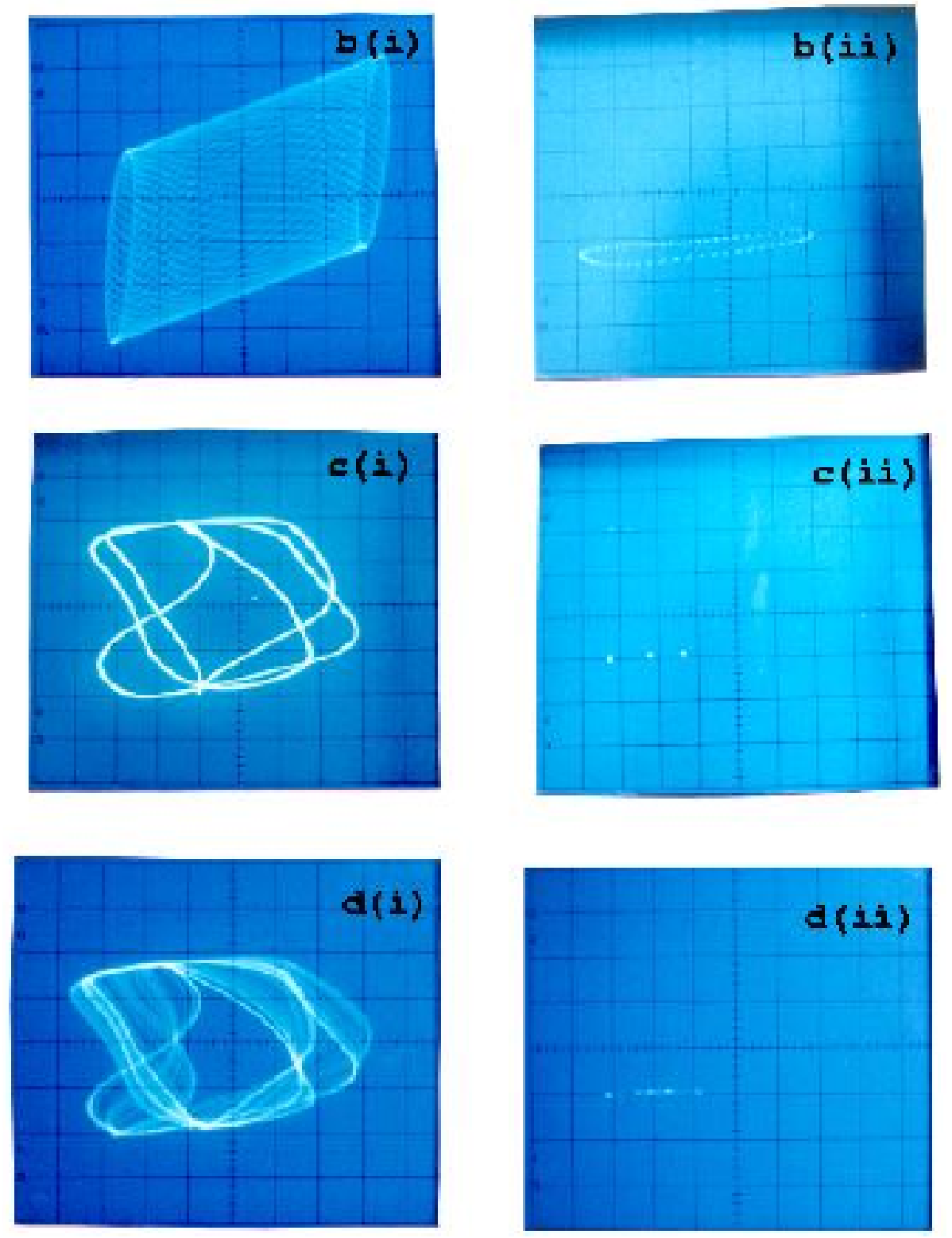

Fig. 12. MLC variant circuit (experimental): (a) Time series plot for voltage $v(t)$ of the limit cycle for $f=0$. (b) - (g) Experimentally observed (i) phase portraits in the $(v-\sin (\Omega t))$ plane and (ii) Poincaré maps in the $\left(v-i_{L}\right)$ plane: (b) quasiperiodic attractor, $f=0.22 \mathrm{~V}$, (c) period-3 window, $f=0.39 \mathrm{~V}$, (d) chaos, $f=0.398 \mathrm{~V}$ (e) period-4, $f=0.405 \mathrm{~V}$, (f) period- $2, f=0.415 \mathrm{~V}$ and $(\mathrm{g})$ period- $1, f=0.421 \mathrm{~V}$. The circuit parameters have been chosen as $C=10.15 \mathrm{nf}, L=445 \mathrm{mH}$, $R=1475 \Omega$ and $\nu=1.116 \mathrm{kHz}$. 

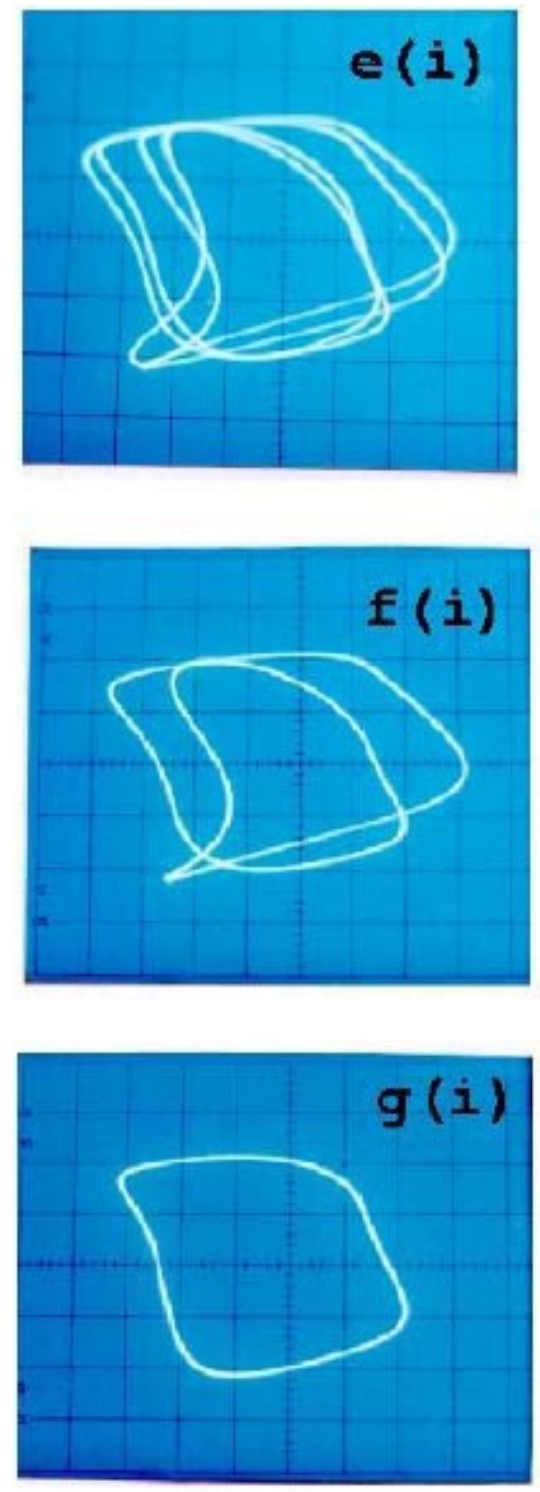

Fig. 12. (Continued)
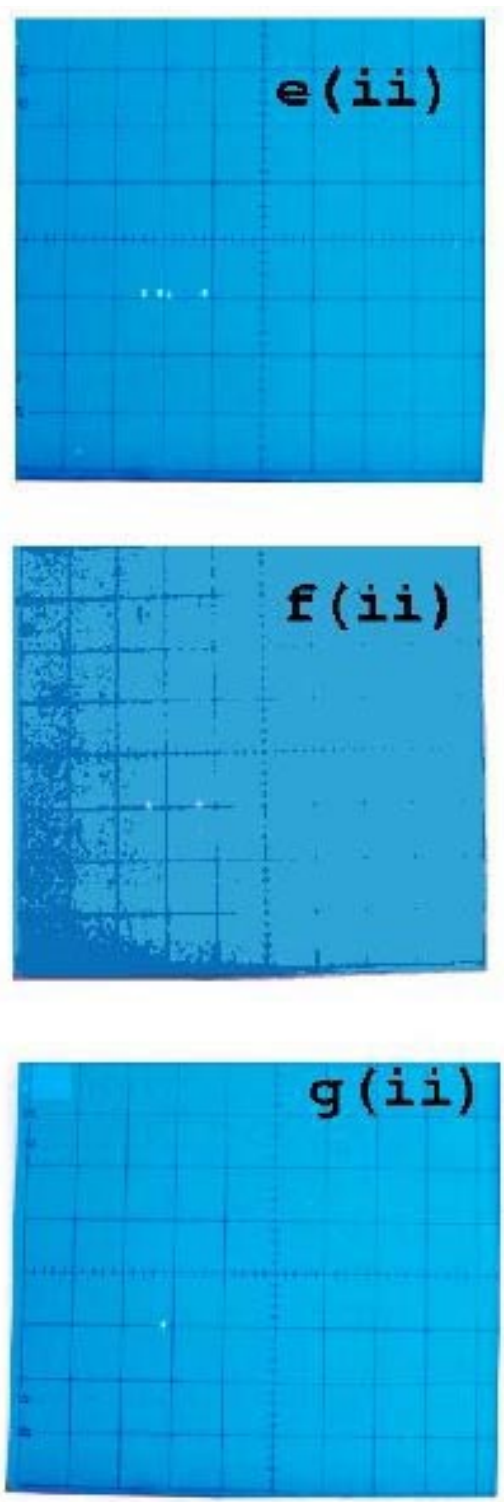

occurs in between the two regular periodic motions in the frequency range $\nu=(1.06 \mathrm{kHz}, 1.15 \mathrm{kHz})$ along the horizontal direction. Figure 12 shows several of the observed attractors as discussed above with respect to the phase diagram (Fig. 11), projected onto the $(v-\sin \Omega t)$ plane along with the Poincaré map of attractors in the order of increasing amplitude $f$ at fixed frequency $\nu=(1.116 \mathrm{kHz})$. To start with for $f=0$, a period-1 limit cycle [Fig. 12(a)] is observed. As already mentioned at $f=0.22 \mathrm{~V}$, we obtain a quasiperiodic (torus) attractor [Fig. 12(b)]. Then at $f=0.391 \mathrm{~V}$, we have a period-3 window [Fig. 12(c)]. If we increase the amplitude $f$ further to $f=0.398 \mathrm{~V}$, we observe a chaotic attractor [Fig. 12(d)]. On 
further increasing $f$, we encounter a reverse perioddoubling sequence: a stable period-4 limit cycle for $f=0.405 \mathrm{~V}$ [Fig. 12(e)], a stable period-2 limit cycle for $f=0.415 \mathrm{~V}$ [Fig. 12(f)], and a period-1 limit cycle for $f=0.421 \mathrm{~V}$ [Fig. 12(g)]. Beyond the period-doubling scenario, boundary crisis is observed for higher driving amplitudes (Fig. 11).

\subsubsection{Intermittency route to chaos}

The phenomenon of intermittency, often observed experimentally [Manneville \& Pomeau, 1980; Schuster, 1988], is characterized by regular (laminar) phases alternating with irregular bursts.
In the present circuit, starting from a fixed amplitude $f=0.32 \mathrm{~V}$ for the frequency in the range $\nu=(1.176 \mathrm{kHz}, 1.182 \mathrm{kHz})$, one finds that a stable period-3 limit cycle disappears through a tangent bifurcation, leading to chaos. However, a section of the period-3 cycle still remains, and the trajectory behaves for most of the time as if it was approaching a period-3 limit cycle, and intermittent behavior is observed when the frequency, $\nu$, is decreased. This is referred to as intermittency of type I. In Fig. 13(ai) the waveform $v(t)$ of a stable period-3 limit cycle for $\nu=1.181 \mathrm{kHz}$ and in Fig. 13(aii) the waveform $v(t)$ of a type I
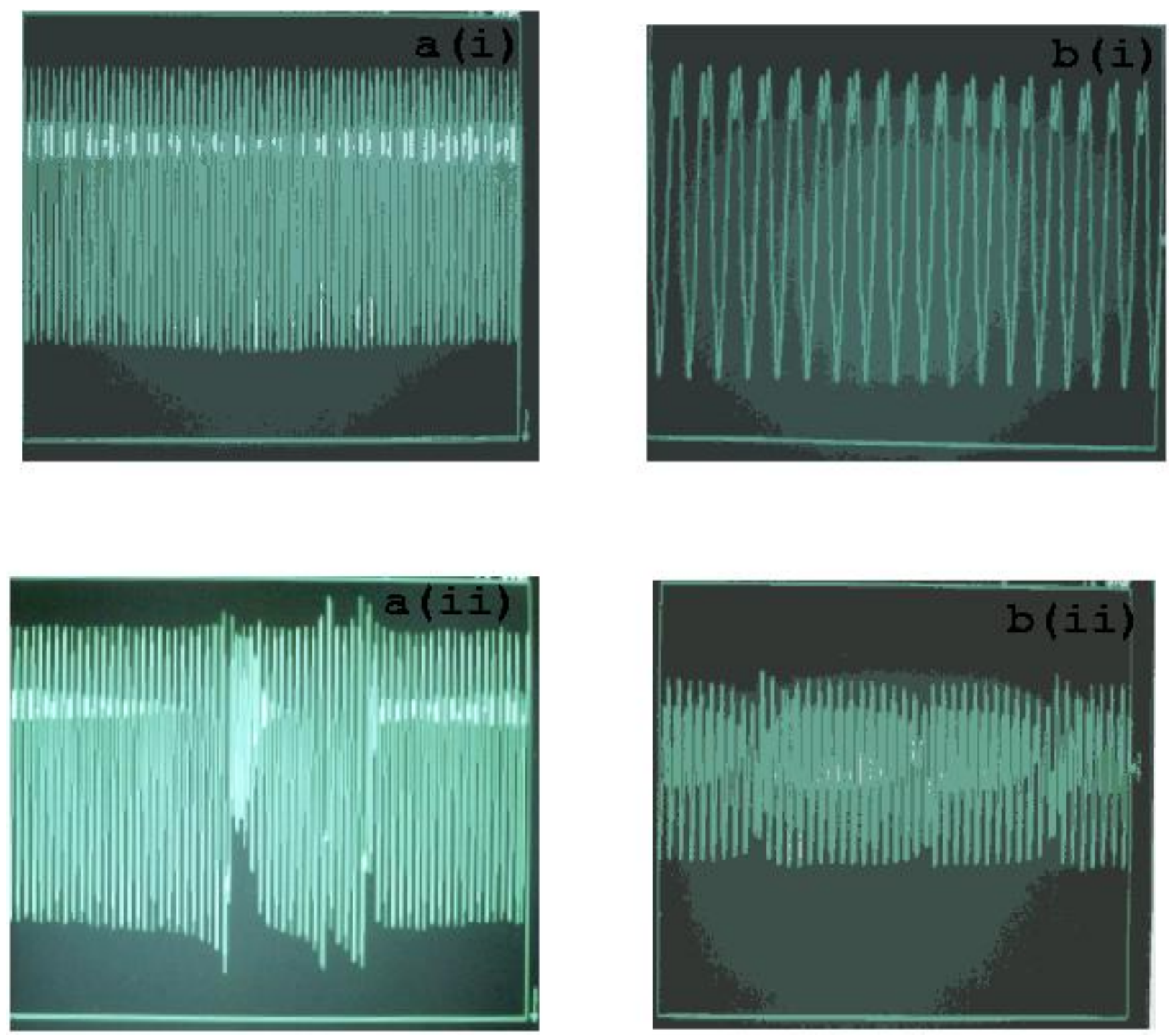

Fig. 13. (a) Type I intermittency near a period-3 window for $f=0.32 \mathrm{~V}$ : (i) the waveform $v(t)$ for a period-3 limit cycle for $\nu=1.181 \mathrm{kHz}$ (ii) the waveform $v(t)$ for an intermittent behavior for $\nu=1.175 \mathrm{kHz}$. (b) Type III intermittency near the boundary crisis regime for $f=0.41 \mathrm{~V}$ and (i) for $\nu=1.101 \mathrm{kHz}$, the waveform $v(t)$ for a period-3 limit cycle and (ii) for $\nu=0.989 \mathrm{kHz}$, intermittent behavior. 
intermittency for $\nu=1.175 \mathrm{kHz}$, with fixed amplitude $f=0.32 \mathrm{~V}$, are shown.

Finally, we have observed type III intermittency route also near the period-doubling bifurcation region beyond the quasiperiodic regime. One such intermittent motion is shown in Fig. 13(b), for a fixed amplitude, $f=0.41 \mathrm{~V}$, for two values of frequency, (i) $\nu=1.101 \mathrm{kHz}$ and (ii) $\nu=989 \mathrm{~Hz}$, showing a transition from period-3 orbit to intermittent behavior, where chaotic bursts appear at a particular subharmonic amplitude together with a decrease (or increase) of the fundamental amplitude. Immediately after this there is a reappearance of the regular (laminar) behavior. This is the characteristic of type III intermittency.

\section{Numerical Analysis of the Variant MLC Circuit}

\subsection{Computer confirmation}

For computer simulation study, we normalize the state equations of the variant of the MLC circuit given in Eq. (3) as in the case of the MLC circuit by appropriate rescaling, $v=x B_{p}, i_{L}=y G B_{p}$, $\omega=\Omega C / G, G=1 / R$ and $t=\tau C / G$ and then redefining $\tau$ as $t$. Consequently, the following set of normalized equations are obtained,

$$
\begin{aligned}
& \dot{x}=F \sin (\omega t)-x-y-g(x), \\
& \dot{y}=\beta x, \quad\left(\cdot=\frac{d}{d t}\right)
\end{aligned}
$$

where $\beta=C / L G^{2}, F=f / B_{p}$. Obviously $g(x)$ is the same piecewise-linear function given in Eq. $(2 \mathrm{c})$, namely

$$
g(x)= \begin{cases}b x+(a-b), & x \geq 1 \\ a x, & |x| \leq 1 \\ b x-(a-b), & x \leq-1\end{cases}
$$

Here $a=G_{a} / G, b=G_{b} / G$. Note that the set of two coupled first-order ordinary differential equations given by Eq. (4) can also be written as a single second-order differential equation of the Lienard's type in the form

$$
\ddot{y}+\dot{y}+\beta g(\dot{y} / \beta)+\beta y=\beta F \sin (\omega t) .
$$

We note at this point that chaos via torus breakdown generated in a piecewise-linear forced van der Pol equation of the form(5) with asymmetric nonlinearity has been studied by Inaba and Mori [1991, 1992] sometime ago. However the corresponding circuit uses more nonlinear elements than the present circuit.

Now the dynamics of Eq. (4) or equivalently (5) depends on the parameters $a, b, \beta, F$, and $\omega$. Then for the chosen experimental circuit parameter values as given in Sec. 3 , we have $\beta=0.05$, $a=-1.121$, and $b=-0.6047$. Once again we consider the dynamics in the $(F-\omega)$ plane either by integrating Eq. (4) or by solving Eq. (5) analytically (see Sec. 4) and numerically.

\subsection{Phase diagram for the $M L C$ variant circuit in the $(F-\omega)$ plane}

In this subsection we will concentrate on a detailed numerical study of Eq. (4) or (5) and confirmation of the experimental results of the variant MLC circuit discussed in Sec. 3. Figure 14 shows the resulting phase diagram in the $(F-\omega)$ plane with fixed values of $\beta, a$ and $b$. The diagram covers the transitions in the region of the external forcing frequency, $0.09 \leq \omega \leq 0.16$, and the forcing amplitude, $0.15 \leq F \leq 0.5$. In this phase diagram also again each colored region denotes a particular type of steady-state behavior, identical to Figs. 2, 5 and 11. Other periodic windows are indicated by the corresponding number (such as $5 T, 6 T$, etc.). One may note that the numerical results are not only in confirmity with the experimental results contained in Fig. 11 but they also correspond to much intricate details.

One observes that Eq. (4) admits a free-running solution when the external periodic signal is absent (corresponding to the autonomous case), see Sec. 5 . When it is present and for low $F$ values and all $\omega$ values in the range of study, the frequency of the system becomes incommensurate with the external frequency. Consequently, the system exhibits two frequency quasiperiodic oscillations in the lower triangular part of the phase diagram, Fig. 14, below the island structure, which extends all the way upto $\omega=0.16$ for low $F$ values. Within the dentritic and island structures, we have observed a series of appearance and disappearance of quasiperiodic, periodic and chaotic attractors involving several interesting bifurcations during the amplitude and frequency scannings. Above the island, in the upper triangular part, we have mostly period-1 attractors. The details are as follows. 


\subsection{Classification of bifurcations and different type of route to chaos}

From the dynamical theory point of view, the phase diagram (Fig. 14) represents several types of bifurcations and routes to chaos: quasiperiodic (torus) route and torus breakdown to chaos, period-adding sequences, Farey sequences, standard period-doubling bifurcations and intermittent routes to chaos, reverse bifurcations, antimonotonicity including remerging Feigenbaum trees and band mergings, to name a few, which we have encountered in our study. These are discussed in the following subsections with the help of suitable oneparameter bifurcation diagrams for either $F$ or $\omega$ scanning along with maximal Lyapunov exponents and phase portraits in the $(x-\sin \omega t)$ plane.

\subsubsection{Quasiperiodic and torus breakdown to chaos}

As was observed in our experimental study, there exists a large portion of the phase space in its lower triangular part where quasiperiodic orbits dominate below the diagonal island with its fine dentritelike structures corresponding to phase-locked oscillations. A typical large region of quasiperiodic oscillations occurs for amplitude range $F=(0.15$, $0.185)$ and frequency range $\omega=(0.09,0.16)$, just below the diagonal island structure. Slightly above this range of $F$, for example at $F=0.225$, one observes a large region of quasiperiodicity interrupted by different phase-locked states before the onset of chaos and then transition to period- 1 orbit, during the $\omega$-scanning. This is depicted in Figs. 15(a) and 15(b). Note the extremely rich fine structure before the onset of chaos and transition to period-1 orbit [Fig. 15(a)]. Similarly at fixed frequency, $\omega=0.145$, as the amplitude $F$ increases $(F \geq 0.15)$ along the upward direction in the amplitude scanning, the quasiperiodic oscillation breaks up and chaotic attractor is obtained at $F \geq$ 0.234 as shown in Fig. 15(b), before period-1 orbit sets in.

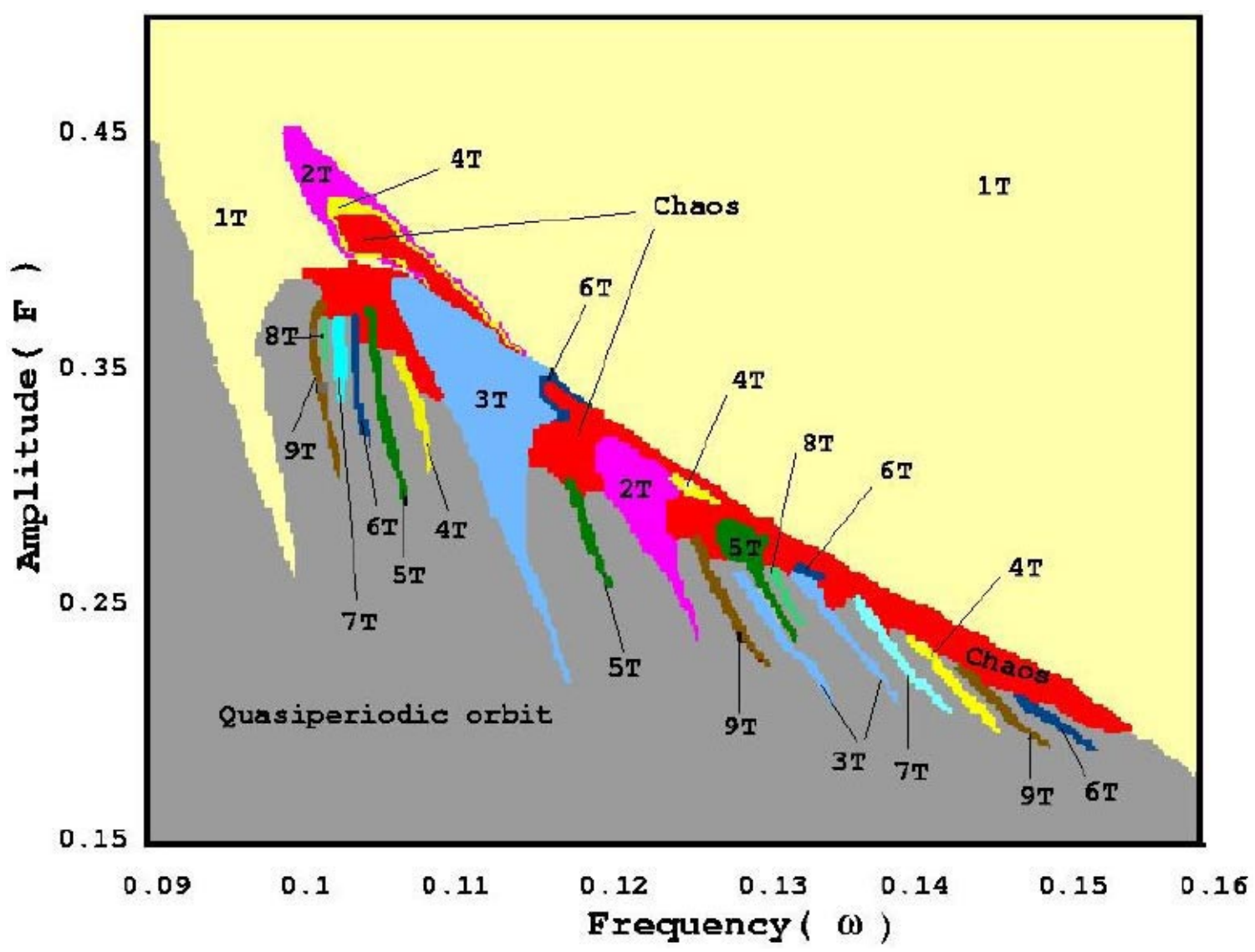

Fig. 14. Computer generated phase diagram in the $(F-\omega)$ plane for the MLC variant circuit, Eqs. (4). Each colored region denotes a particular type of steady-state behavior as in Figs. 2, 5 and 11. 

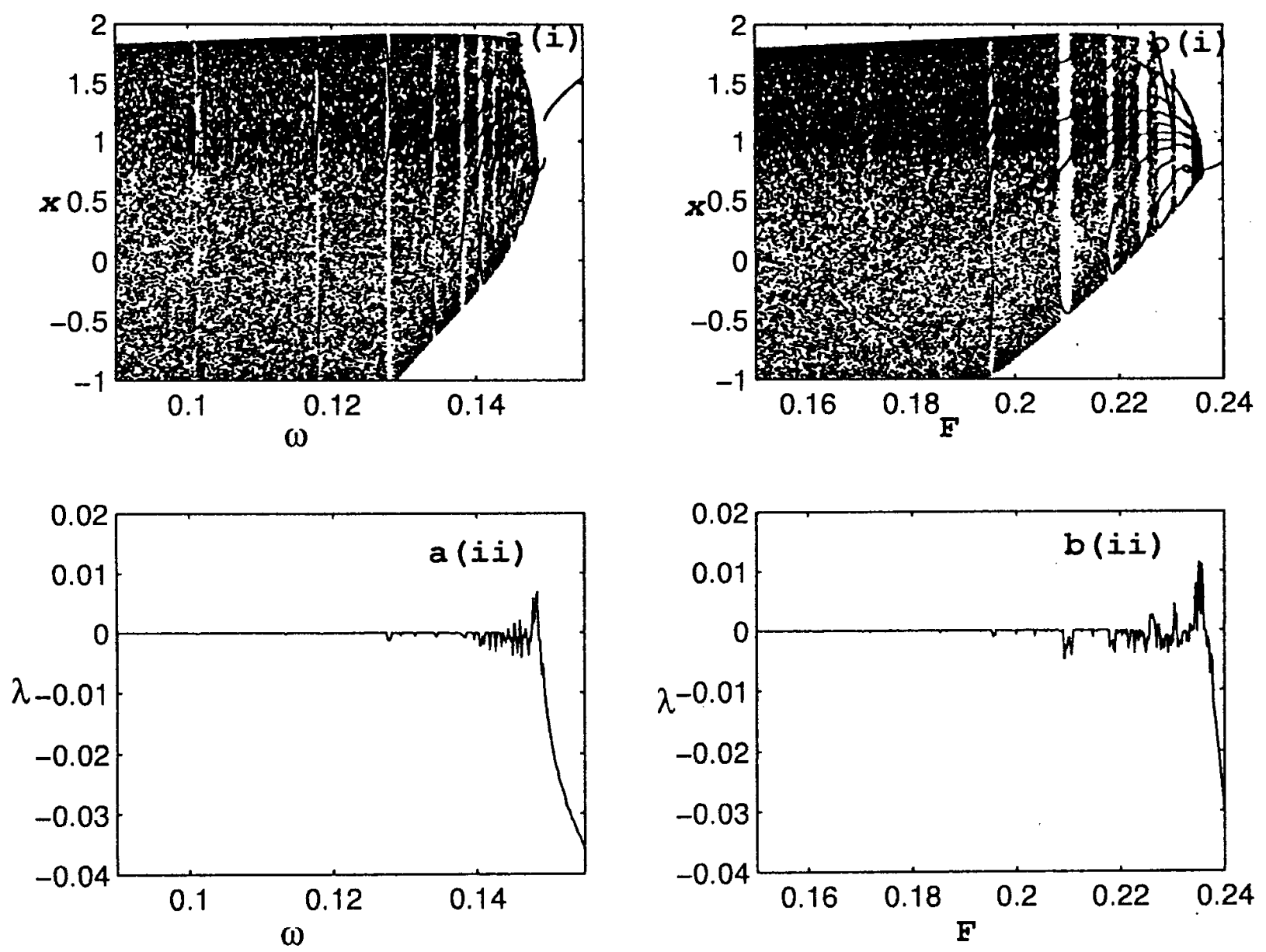

Fig. 15. The transition from quasiperiodic attractor to chaos: (ai) Frequency scanning in Fig. 14- one-parameter bifurcation diagram in the $(\omega-x)$ plane for fixed $F=0.225$ and (aii) maximal Lyapunov exponent of (i). (bi) Amplitude scanning in Fig. 14 - one-parameter bifurcation diagram in the $(F-x)$ plane for fixed $\omega=0.145$ and (bii) maximal Lyapunov exponent of (i).

\subsubsection{Period-adding sequences}

Corroborating our experimental study of the present circuit, our numerical analysis confirms the phenomenon of period-adding sequences, where windows of consecutive periods are separated by regions of quasiperiodicity, particularly in the dentritic regions and during torus breakdown to chaos. In other words, for a fixed value of $F$, we obtain a stable period- $n$ orbit, $n=1,2,3, \ldots$, followed by a region of quasiperiodicity (torus), then a stable period $-(n+1)$ orbit and so on. In the computer generated phase diagram, we have identified a large sequence of periodic windows of several different orders, starting from period-3 to period-9 in the middle amplitude and low frequency region in Fig. 14. For example, at higher drive amplitude $F=0.352$ and narrow lower frequency range $\omega=(0.101,0.1095)$, we have observed win- dows of periods $3,4,5,6,7,8,9$ with intervening quasiperiodic and other orbits as $\omega$ decreases along the horizontal direction [see Fig. 16(a)]. Here, the transition from one quasiperiodic state to another is initiated by a period-adding bifurcation followed by recovery to the next periodic state and so on before entering into the chaotic region. We have identified many other period-adding sequences during both $F$ and $\omega$ scannings.

\subsubsection{Farey sequences}

Within the period-adding sequences, if we look at Fig. 16(a) carefully, we also find that periods of some of the windows satisfy the familiar Farey sequence: For example, between period-6 and period- 5 windows there exists a period-11 $(=6+5)$ window in the region $\omega=(0.104,0.105)$ and between period-9 and period-4 windows there is a 

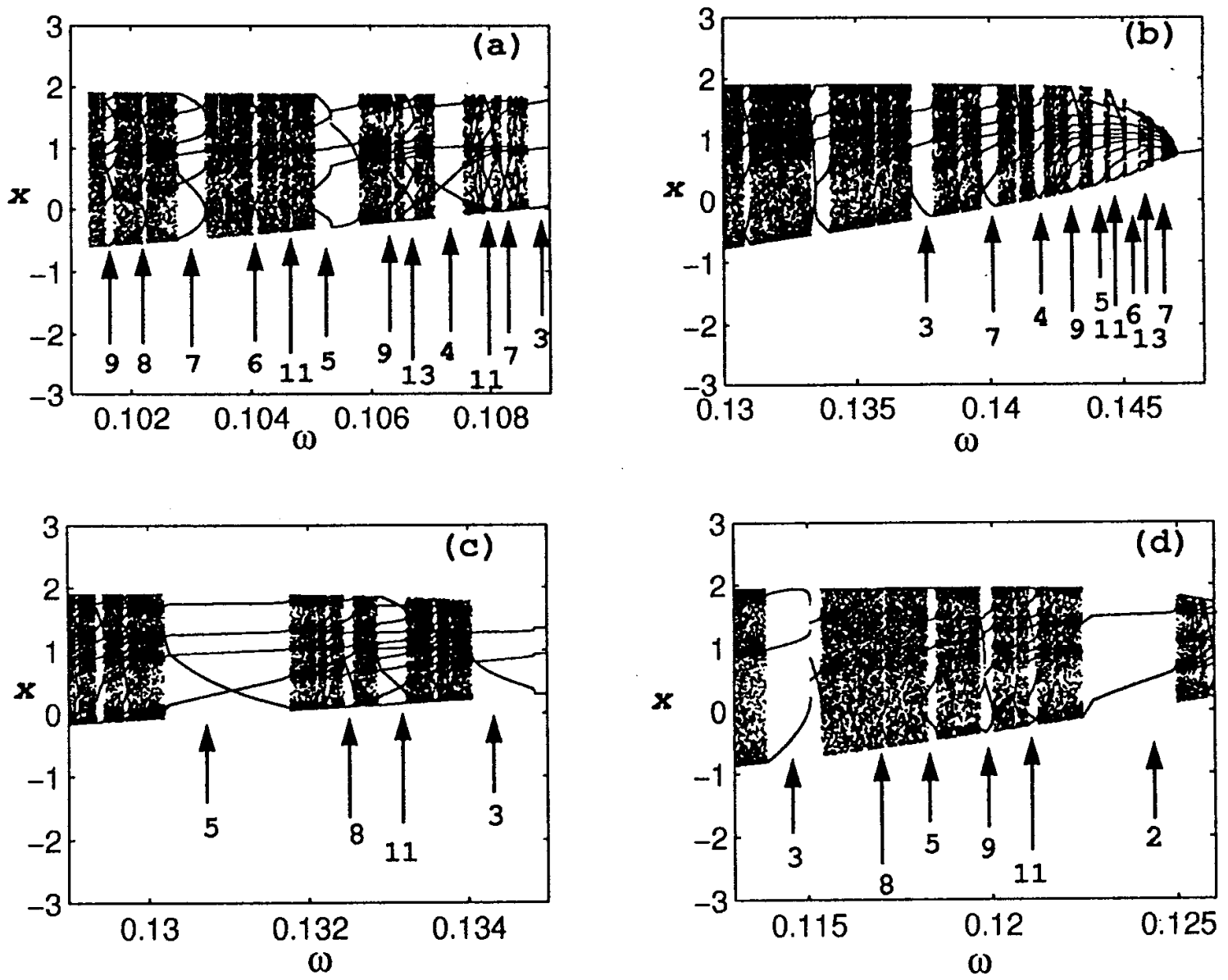

Fig. 16. Bifurcation diagram for frequency scanning in the $(\omega-x)$ plane illustrating period-adding sequences at fixed (a) $F=0.352$ and Farey sequences, (b) $F=0.23$, (c) $F=0.265$, and (d) $F=0.28$.

period-13 $(=9+4)$ window in the region $\omega=(0.106$, $0.1075)$, and between period- 4 and period-7 window there is a period-11 $(=4+7)$ window in the region $\omega=(0.1075,0.1085)$ and so on.

In addition to the above, there are several other prominent regions exhibiting Farey sequences in the phase diagram, Fig. 14. Some of them are listed below.

(i) As the forcing parameter $\omega$ increases in the range $\omega=(0.11,0.145)$ and at different fixed amplitude $(F)$ values, there are several situations where we find that in between period $-(n)$ window and $-(n+1)$ window, there is a phase-locked window of period $(2 n+1)$. In the phase diagram, Fig. 14, in the region $F=(0.23,0.24)$, and $\omega=(0.13,0.145)$, between $3 T$ and $4 T$ window regimes, there is a $7 T$ window $(=3+4)$. On a finer scale, as may be seen from the one-parameter bifurcation diagram in Fig. 16(b) for a fixed $F=0.23$, between $3 T$ and $4 T$ there is a $7 T(=3+4)$ window, between $4 T$ and
$5 T$ there is a $9 T(=4+5)$ window and between $5 T$ and $6 T$ there is a $11 T(=5+6)$ periodic orbit. Also, a period $13 T$ window $(=6+7)$ appears before the chaos region between $6 T$ and $7 T$ windows [see Fig. 16(b)].

(ii) Further at the amplitude $F=0.265$ within the narrow frequency range $\omega=(0.130,0.135)$ in the quasiperiodic region between $5 T$ and $3 T$ windows, there is a $8 T$ window $(=5+3)$ and between $8 T$ and $3 T$ there is a $11 T$ window $(=8+3)$ [see Fig. 16(c)], and so on.

(iii) Also, at $F=0.28$ within the frequency region $\omega=(0.11,0.13)$ in between $3 T$ and $2 T$ window regions there is a $5 T$ window $(=3+2)$ and on a finer scale between the $3 T$ and $5 T$ window regions, there is an $8 T$ window $(=3+5)$ and so on [see Fig. 16(d)].

\subsubsection{Period-doubling and reverse period-doubling bifurcations}

Besides the cascade of period-adding and Farey 
sequences, the standard period-doubling bifurcation sequences and chaotic attractors followed by periodic windows have been observed in the regions of higher drive amplitude $(F)$ and certain regions of low frequency $(\omega)$ values. For example, at fixed frequency $\omega=0.105$ on decreasing the amplitude $F$ in the range $F=(0.4,0.43)$ along the downward direction in the amplitude scanning, period-doubling bifurcations via chaos occur - see the phase diagram (Fig. 14). Figure 17(ai) shows the one-parameter bifurcation diagram in the $F-x$ plane, which clearly indicates the familiar period-doubling bifurcation sequence, chaos, windows, etc. as $F$ is decreased. In Fig. 17(aii) the corresponding maximal Lyapunov exponent is plotted. Similarly, as an example, by making an $\omega$-scanning for fixed $F=0.388$, in the range $\omega=(0.105,0.106)$, one can observe the perioddoubling route to chaos [Fig. $17(\mathrm{~b})]$ as $\omega$ is increased.

In addition, beyond the period-doubling cascades, we observe reverse period-doubling bifurcations at higher drive amplitude, $F$, and at lower frequency, $\omega$, upon increasing or decreasing $F$ and $\omega$ values as the case may be (see the phase diagram (Fig. 14)). For example at fixed amplitude $F=0.395$, on increasing the frequency $\omega$ in the range $\omega=(0.104,0.1105)$ along the horizontal direction [Fig. 17(c)] and at fixed frequency $\omega=0.1043$, on increasing the amplitude, $F$ in the range $F=(0.388,0.43)$ along the vertical direction [Fig. 17(d)] one can identify period-doubling sequences leading to chaos, window, chaos, and then reverse period-doubled orbits.
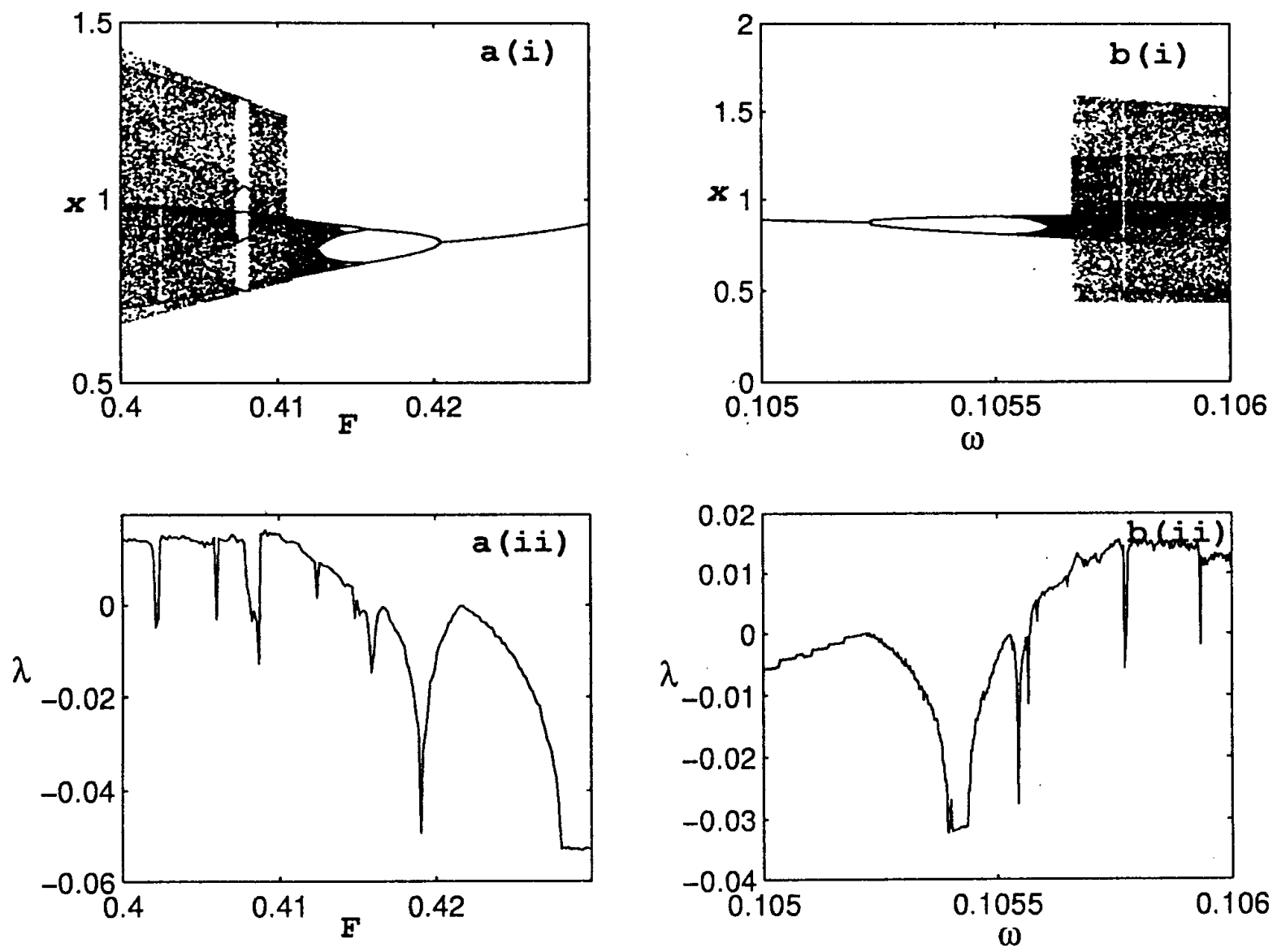

Fig. 17. Period-doubling bifurcations: (ai) one-parameter bifurcation diagram in the $(F-x)$ plane and (aii) maximal Lyapunov exponent in the $\left(F-\lambda_{\max }\right)$ plane. The value of frequency has been fixed at $\omega=0.105$ in the amplitude scanning. (bi) One-parameter bifurcation diagram in the $(\omega-x)$ plane and (bii) maximal Lyapunov exponent in the $\left(\omega-\lambda_{\max }\right)$ plane. The value of frequency has been fixed at $F=0.388$ in the frequency scanning. Combined period-doubling and reverse period-doubling phenomenon: (ci) one-parameter bifurcation diagram - frequency scanning in the $(\omega-x)$ plane for fixed $F=0.395$ and (aii) maximal Lyapunov exponent of (i). (di) Amplitude scanning in the $(F-x)$ plane for fixed $\omega=0.1043$ and (dii) maximal Lyapunov exponent of (i). 

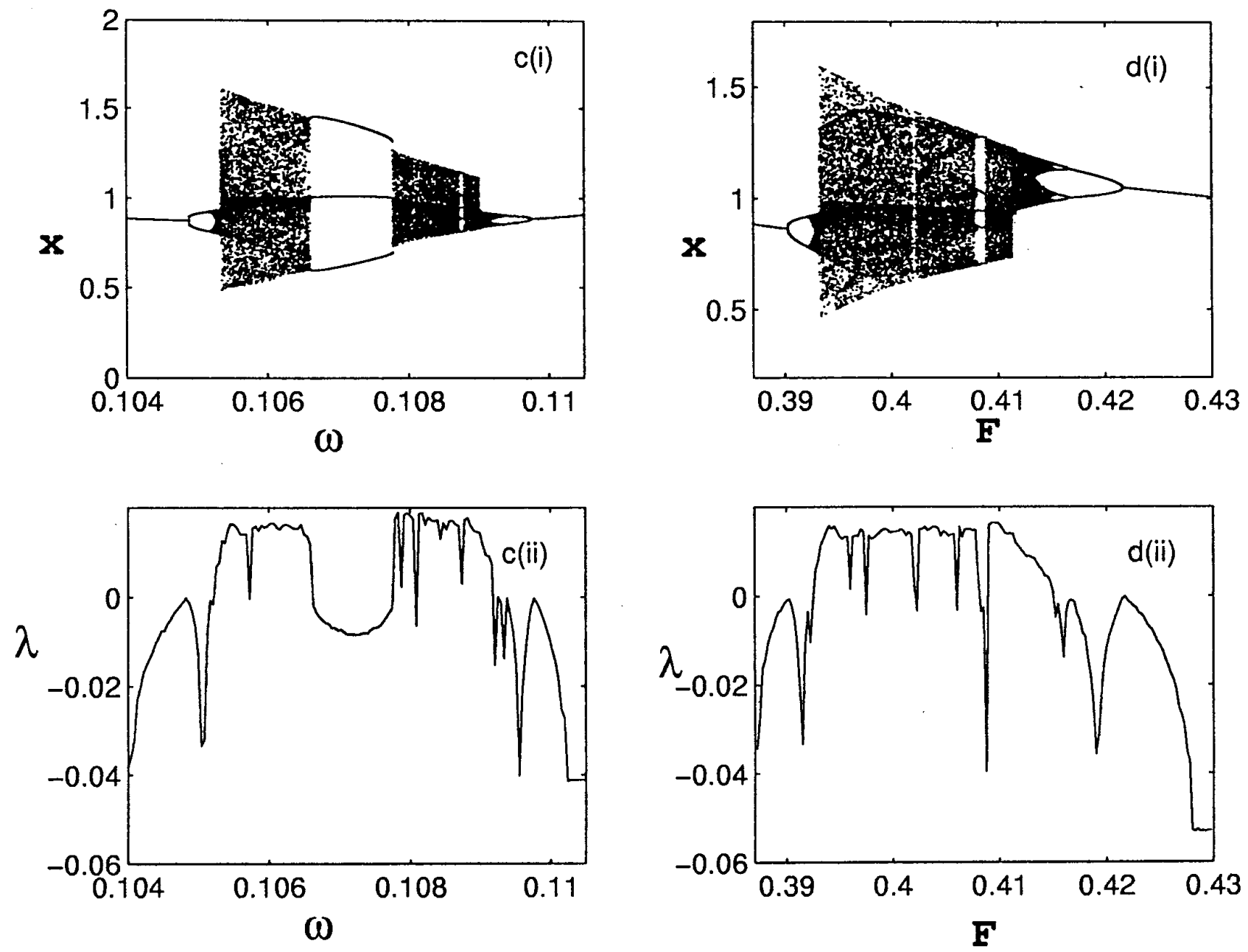

Fig. 17. (Continued)

In the earlier section, we have displayed typical phase portraits of some of the basic attractors obtained through period-doubling bifurcations and quasiperiodicity [Fig. 12]. Figure 18 is the counterpart of Fig. 12 by computer generated phase portrait in the $(x-\sin \omega t)$ plane along with the corresponding Poincaré maps. We have also calculated the Lyapunov exponents for various values of $F$ to confirm the nature of the attractors.

\subsubsection{Remerging Feigenbaum trees, bubbling, band merging and antimonotonicity}

As pointed out in Sec. 2, finite period-doubling sequences [Bier \& Bountis, 1984; Kypriandis et al., 2000] "merging" as it were with inversely advancing ones can arise to form a finite number of "bubbles" on some cross-sections of the full parameter space, leading to the property of antimonotonicity. An important consequence of such a remerging is that low-order periodic orbits become again stable and relatively large regions reappear around them, where the motion is regular and predictable [Bier \& Bountis, 1984]. Such remerging period-doubling sequences (or Feigenbaum trees) commonly arise in some of the simplest nonlinear dynamical systems involving the variation of more than one parameter. In the present study, we find that remerging Feigenbaum trees exist at higher drive amplitude and low frequency values in the ranges $F=(0.4,0.43)$ and $\omega=(0.1,0.107)$ in the parameteric space. For example, for $F=0.425$ in the frequency range $\omega=(0.1,0.106)$ a period-2 bubble is present [Fig. 19(a)] and the branch develops a stable period-4 bubble at $F=0.422$ [Fig. 19(b)]. On the other hand at $F=0.4205$ in the same frequency range, we have period-8 bubble [Fig. 19(c)]. Similarly at $F=0.42033$, we have a period- 16 bubble [Fig. 19(d)]. As $F$ is decreased further, more 
bubbles are created until an infinitely branched tree (like chaos) finally appears at $F=0.4198$ as in Fig. 19(e).

Interestingly, as the frequency $\omega$ is increased in the range $\omega=(0.101,0.107)$ [Figs. 19(f)-19(i)] we also observe that the two bands of the chaotic attractor merge into a single band when the amplitude, $F$, is gradually decreased beyond $F=0.4198$. In Fig. 20(a), we can see that for fixed $F=0.413$ when $\omega$ is increased through $\omega=0.10328$, chaotic bands start to merge into a larger one.
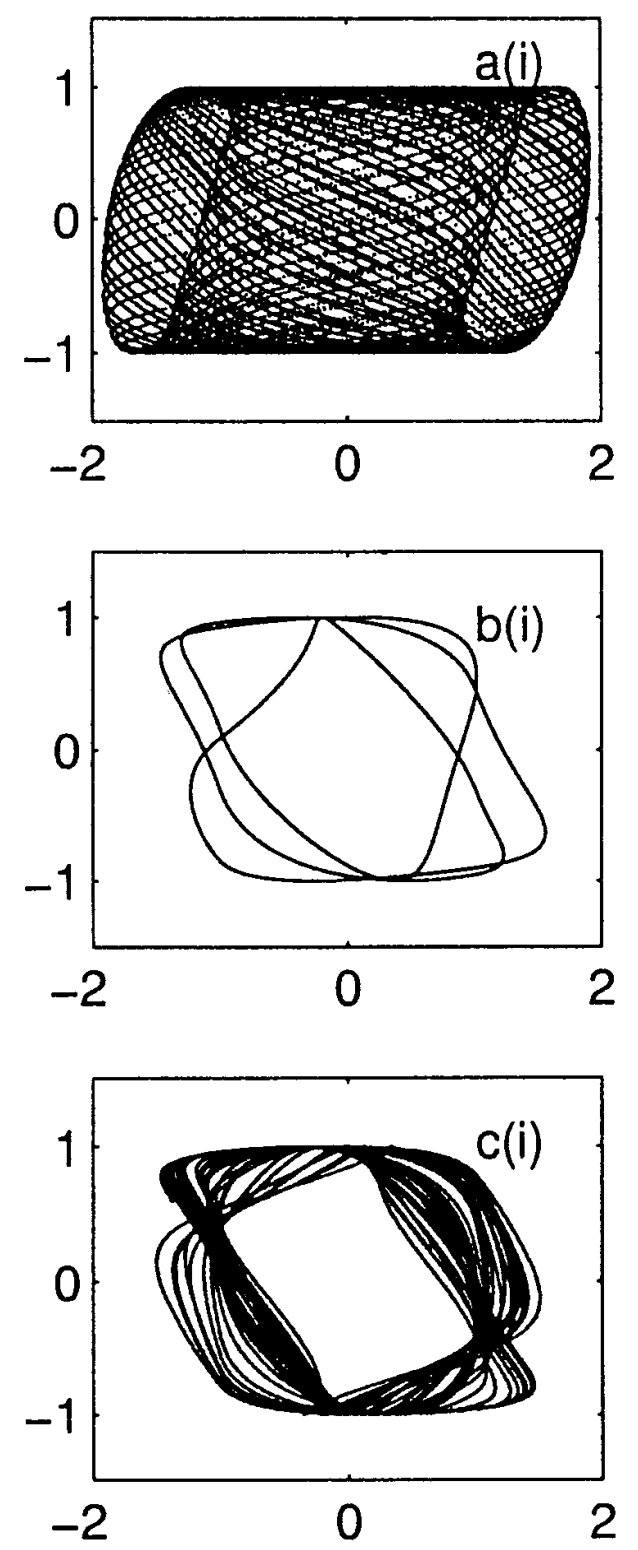

Another interesting example of antimonotonicity has been observed by looking at the phase diagram at the fixed amplitude $F=0.4085$ and varying the frequency in the range $\omega=(0.1035$, 0.1055). Here a tangent bifurcation of the periodic orbit with period- 5 is followed by a complete sequence of period-doubling bifurcations; then after an interval with chaotic behavior, there is a reverse period-doubling sequence, ending in a periodic orbit with period-5 which ultimately undergoes another tangent bifurcation to chaos. This pattern
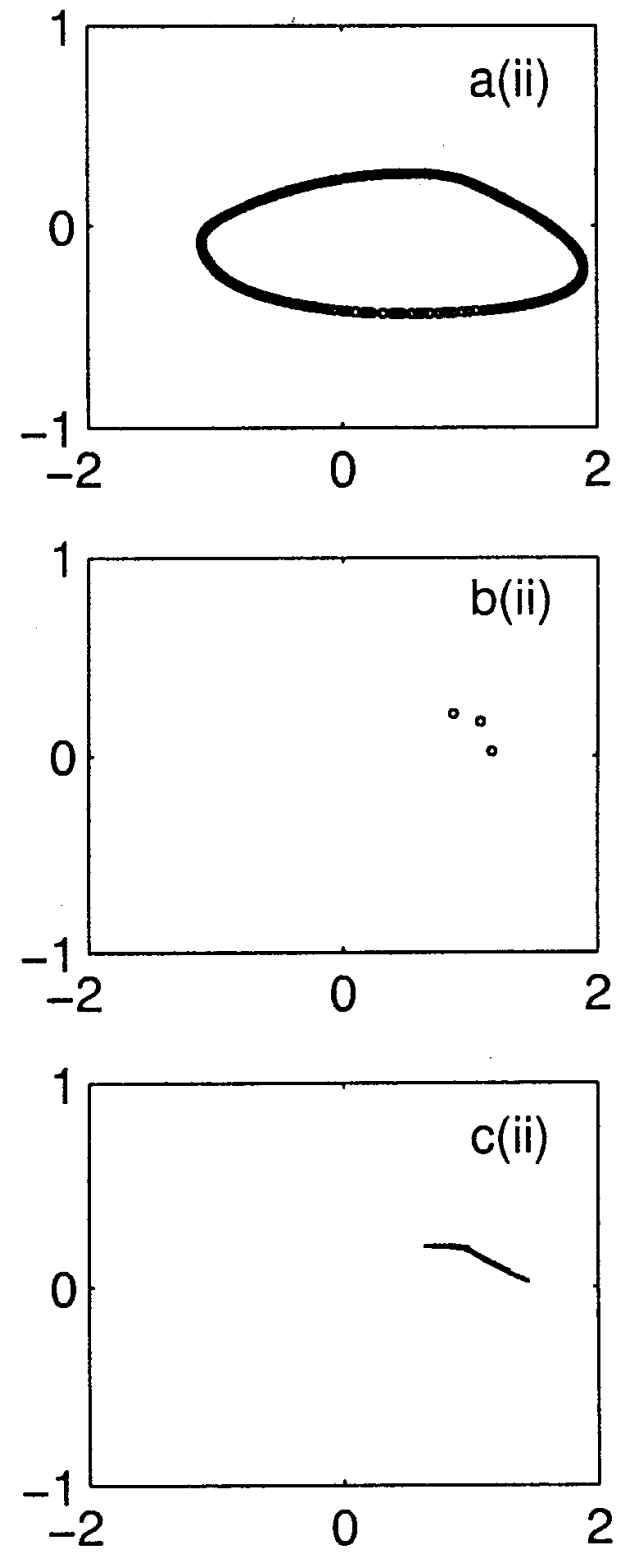

Fig. 18. Computer generated (i) phase portraits in the $(x-\sin \omega t)$ plane of Eq. (4) and (ii) Poincaré map plots in the $(x-y)$ plane corresponding to the parameters $\beta=0.05, \omega=0.105, a=-1.121$ and $b=-0.6047$. (a) $F=0.25$, quasiperiodic motion (b) $F=0.394$, period-3 window (c) $F=0.400$, chaotic attractor (d) $F=0.415$, period-4 limit cycle (e) $F=0.417$, period-2 limit cycle (f) $F=0.431$, period-1 limit cycle. 

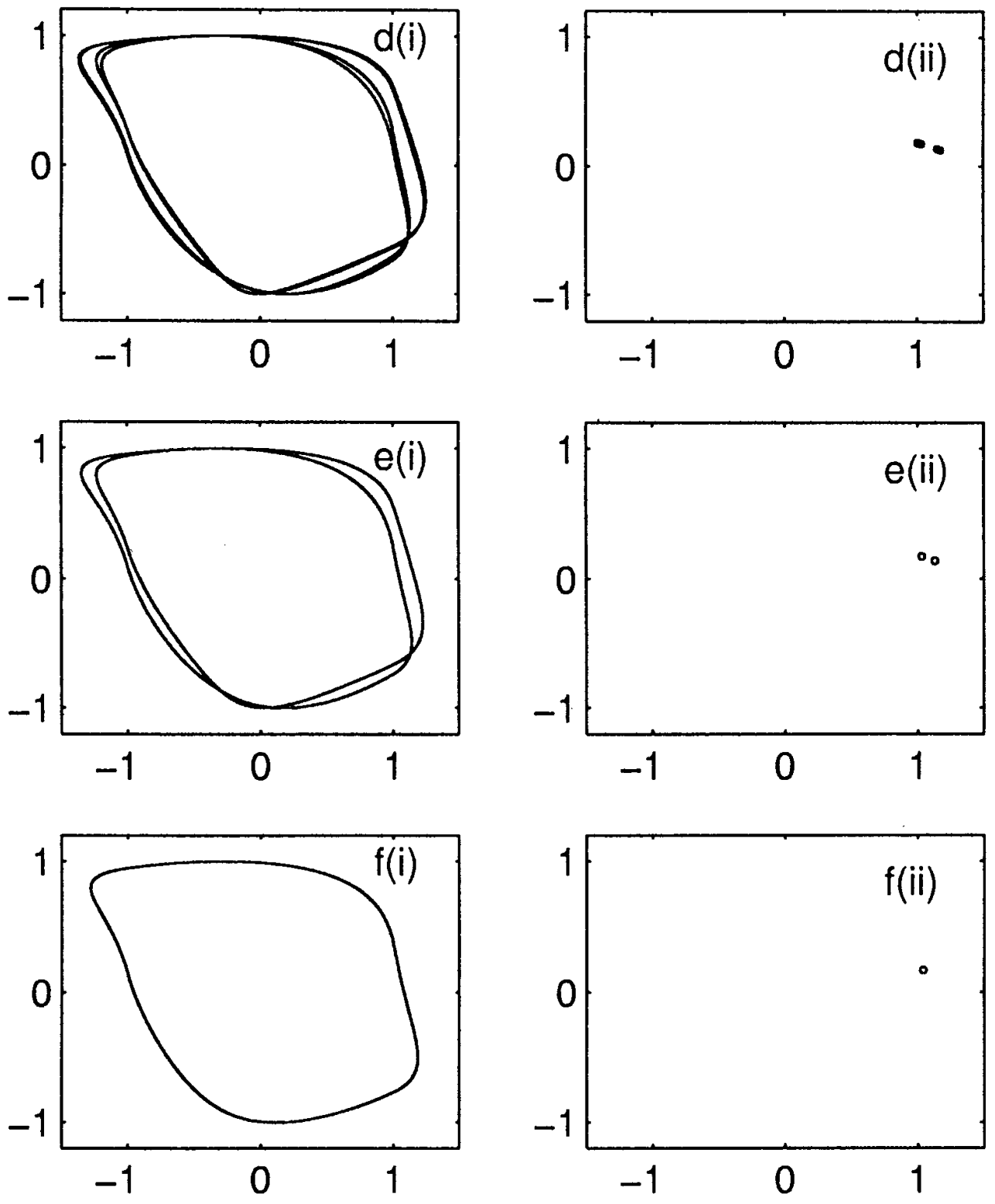

Fig. 18. (Continued)

is obviously a period-5 bubble and it is shown in Fig. 20(b).

\subsubsection{Intermittency routes to chaos}

We have observed that the system (4) or (5) also admits the intermittency routes to chaos for suitable ranges of parameters, as expected from experimental results. For example, we have observed that for $F=0.32$ and $\omega$ in the range $\omega=(0.11125$, 0.11128) type I intermittency occurs through a transition from period-3 window to chaos via intermittency (type I) across the saddle-node boundary $(\omega=0.11125)$. The intermittency signature is shown in Fig. 21(a), where the periodic oscillations are interrupted by intermittent amplitude bursts in the range $\omega=(0.11125,0.11128)$ as $\omega$ is decreased. With further decrease in the frequency $\omega$, the system gives birth to fully developed chaos. The average laminar length $(\langle l\rangle)$ during this transition is found to comply with the law $(\langle l\rangle) \sim \varepsilon^{-\alpha}$, with $\alpha=0.6$, where $\varepsilon=\left(\omega_{c}-\omega\right)$ and $\omega_{c}$ is the bifurcation threshold.

In addition, we have also confirmed the existence of type III intermittency in this system when subcritical period-doubling bifurcation occurs. In the present case, at fixed amplitude, $F$, the transition from period- 8 attractor to period- 4 

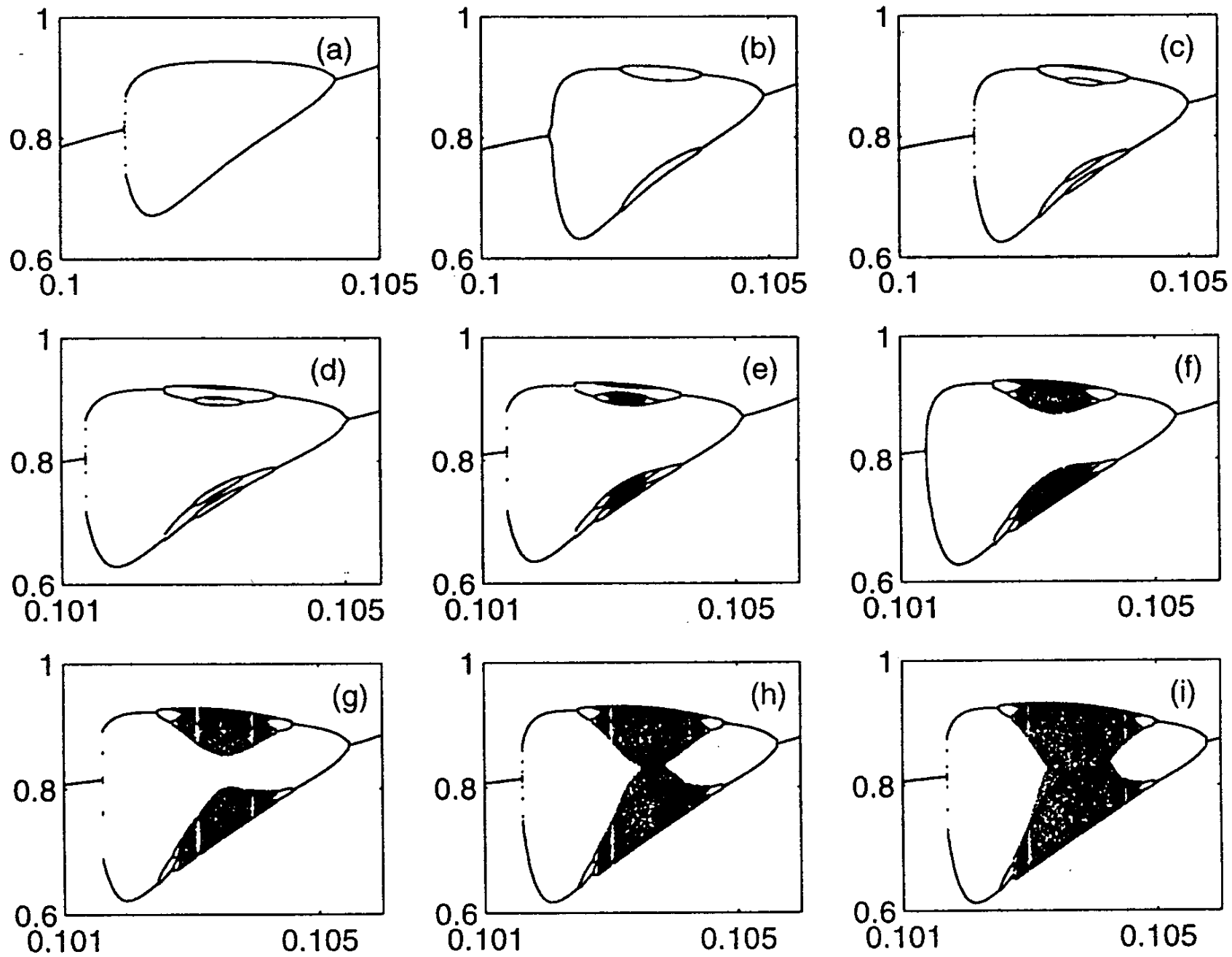

Fig. 19. Bifurcation diagram for frequency scanning in the $(\omega-x)$ plane for remerging Feigenbaum tree (bubbling) (a) The primary bubble at $F=0.425$, (b) period- 4 bubble at $F=0.422$, (c) period- 8 bubble at $F=0.4205$, (d) period-16 bubble at $F=0.42033$ (e) infinite period bubble at $F=0.4198$ and full Feigenbaum remerging tree at $(f) F=0.4185,(\mathrm{~g}) F=0.4175$, (h) $F=0.4165$ and (i) $F=0.4155$.

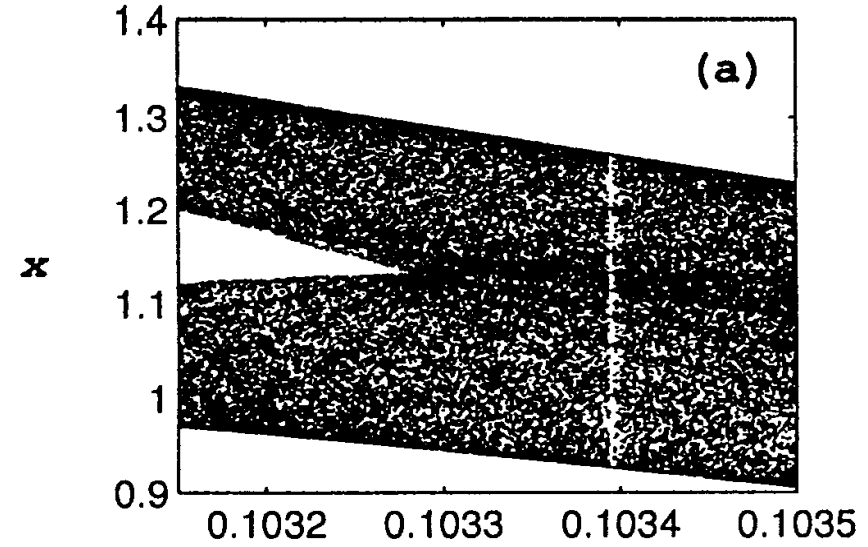

$\omega$

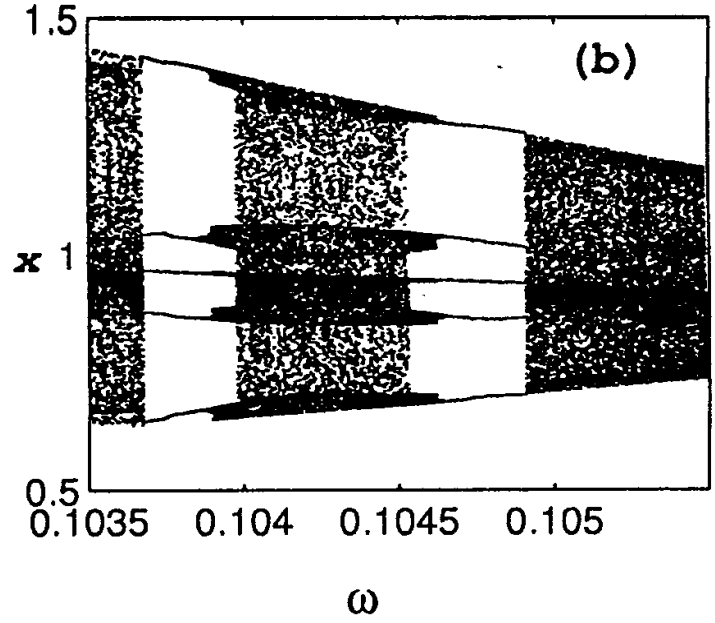

$\omega$

Fig. 20. Bifurcation diagram in the $(\omega-x)$ plane corresponding (a) band merging at fixed $F=0.413$ and $\omega=(0.1055,0.107)$,

(b) period-5 bubble at fixed $F=0.4085$ and $\omega=(0.1035,0.1055)$. 

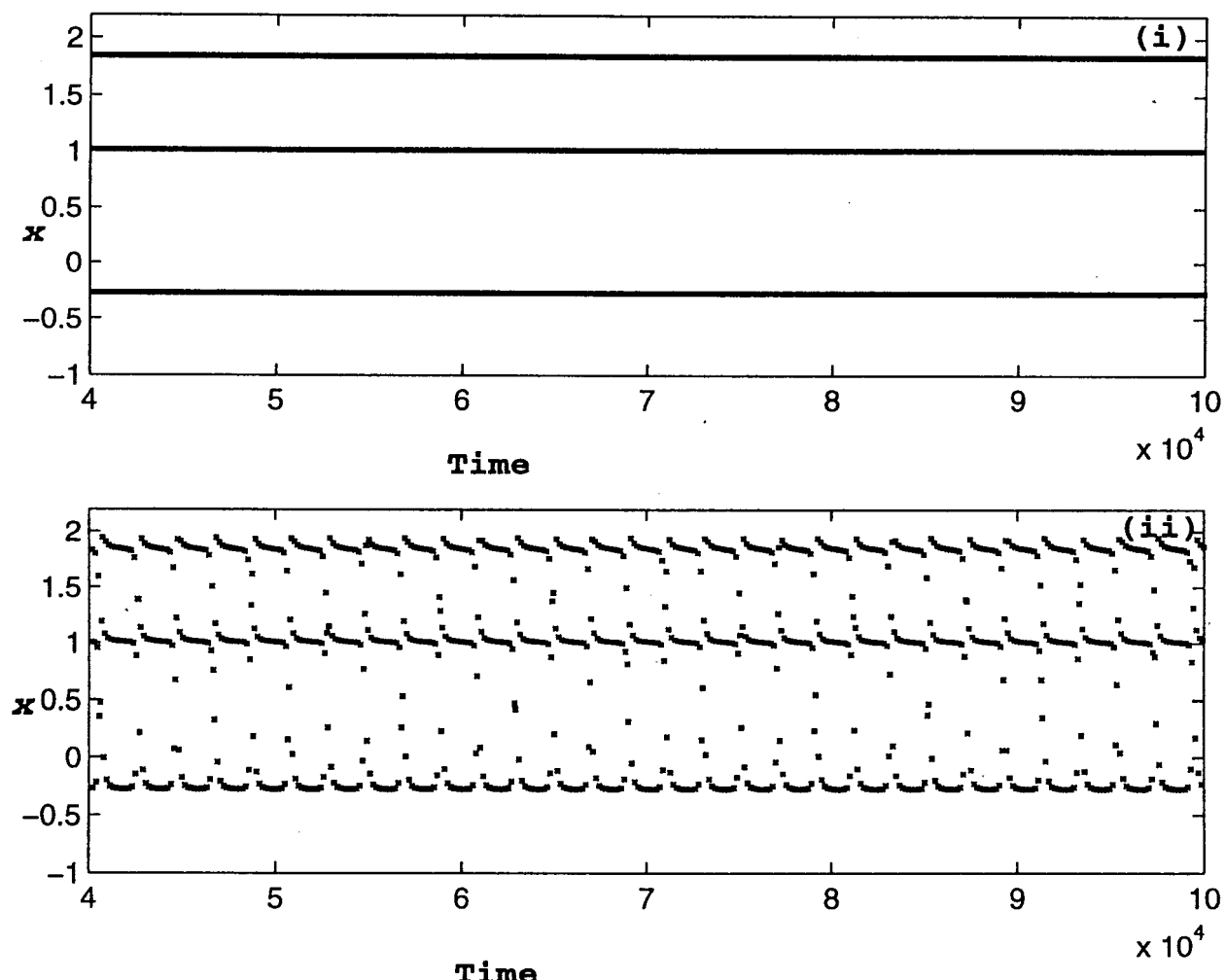

(a)
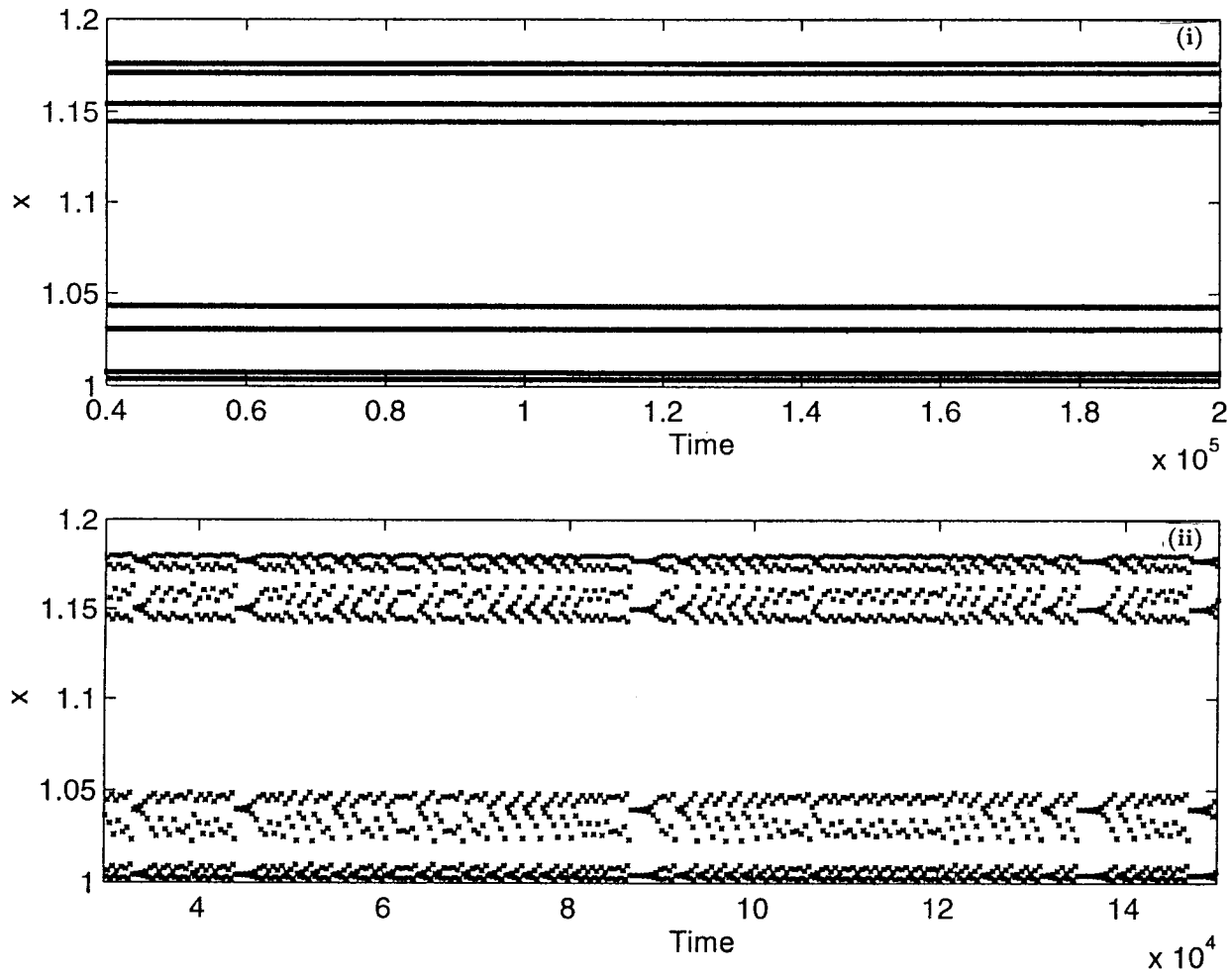

(b)

Fig. 21. (a) Signature of type I intermittency: Poincaré plot for $F=0.32$ and (i) $\omega=0.11128$, and (ii) $\omega=0.11125$.

(b) Signature of type III intermittency: Poincaré plot for $F=0.414$ and (i) $\omega=0.104825$ and (ii) $\omega=0.10482$. 
attractor occurs through subcritical period-halving bifurcation when the frequency, $\omega$, decreases in the range $\omega=(0.1052,0.10482)$. The predominant signature in this transition is emerging through intermittency. One such intermittency motion is shown in Fig. 21(b) which are the Poincaré plots for $F=0.414$ and $\omega=0.104825$ [Fig. 21(bi)] and $\omega=0.10482$ [Fig. 21(bii)]. To confirm further whether the intermittency nature is of type III, we have calculated the average length $(\langle l\rangle)$ of the laminar phase of this intermittency which complies with the following scaling law predicted by Pomeau and Manneville: $\langle l\rangle \sim(1 / \varepsilon)^{\beta}$, with $\beta=0.93$ where $\varepsilon=\omega_{c}-\omega$ and $\omega_{c}$ is the bifurcation threshold.

\subsubsection{Neimark bifurcations}

When the value of the external periodic signal $F$ exceeds a certain critical value during the amplitude scanning for fixed low values of $\omega$, in the region $\omega=(0.09,0.1)$, a transition from periodic motion to quasiperiodic occurs essentially due to supercritical
Neimark bifurcation [Venkatesan \& Lakshmanan, 1997] when $F$ is decreased. This phenomenon also occurs in the region of $\omega$ exceeding the value 0.1545 for low values of $F(F<0.215)$.

\section{SPICE Simulation}

In recent years, circuit simulations such as SPICE [Roberts \& Sedra, 1997] have been used for the simulation of the dynamics of nonlinear circuits. We have simulated the behavior of the MLC and variant MLC circuits for specific circuit parameters. In Fig. 22, we have presented the chaotic attractors evaluated through PSPICE corresponding to $f=0.138 \mathrm{~V}$ in Fig. 3(ei) of the MLC circuit and $f=0.398 \mathrm{~V}$ in Fig. 12(di) of the MLC variant experimental circuit. The corresponding numerical simulation for amplitude $F=0.128$ in Eq. (2) and $F=0.4 \mathrm{~V}$ in Eq. (4) have already been given in Figs. 9(e) and 18(ci), respectively. Similarly we have verified the dynamical behavior for several other values of $\nu$ and $f$ of the MLC and variant MLC circuits given in Figs. 1 and 10.

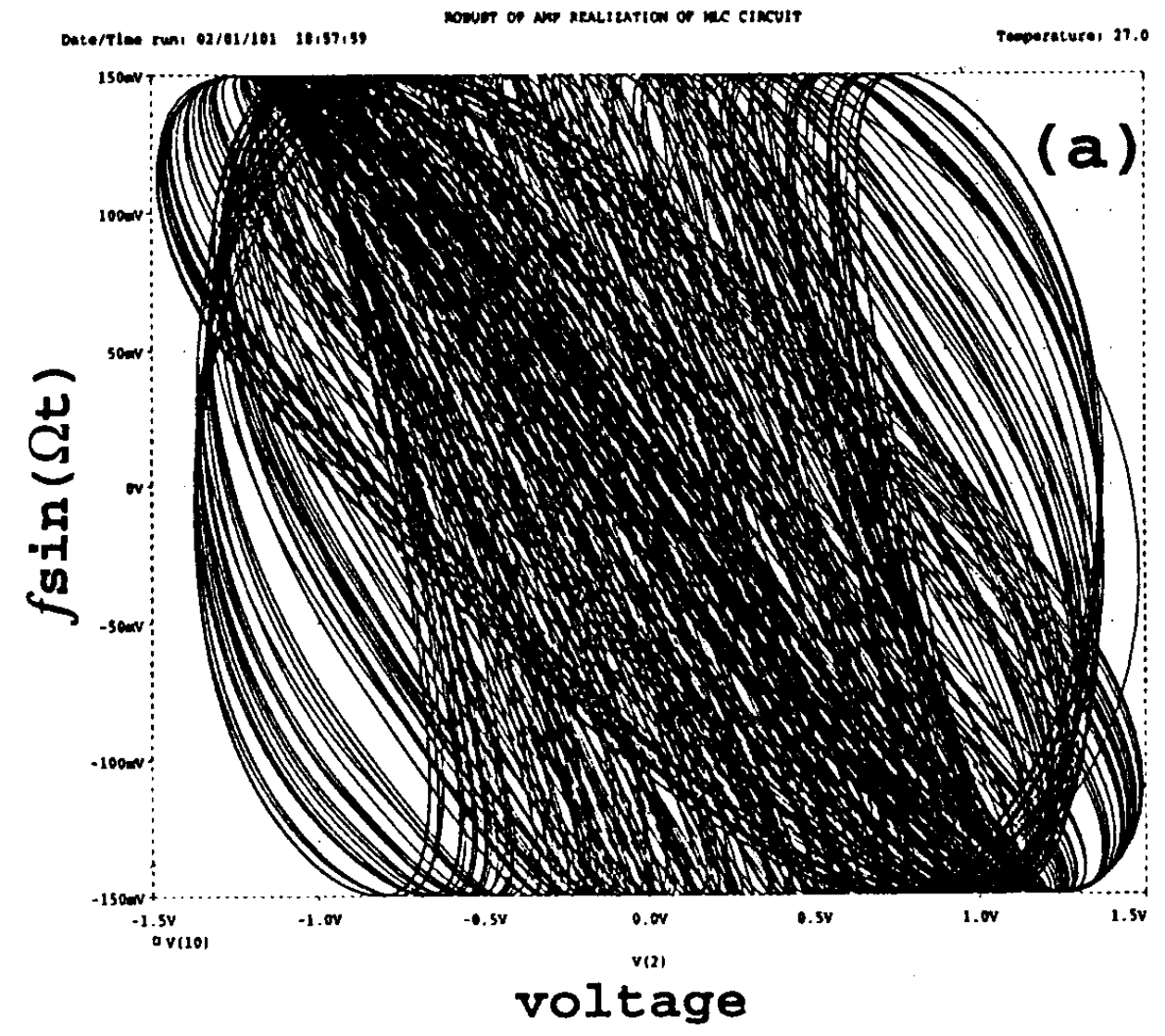

Fig. 22. PSPICE simulation for confirmation of the chaotic attractor (a) the MLC circuit given in Figs. 3(ei) and 9(e), and (b) the MLC variant circuit given in Figs. 12(di) and Fig. 18(ci). 


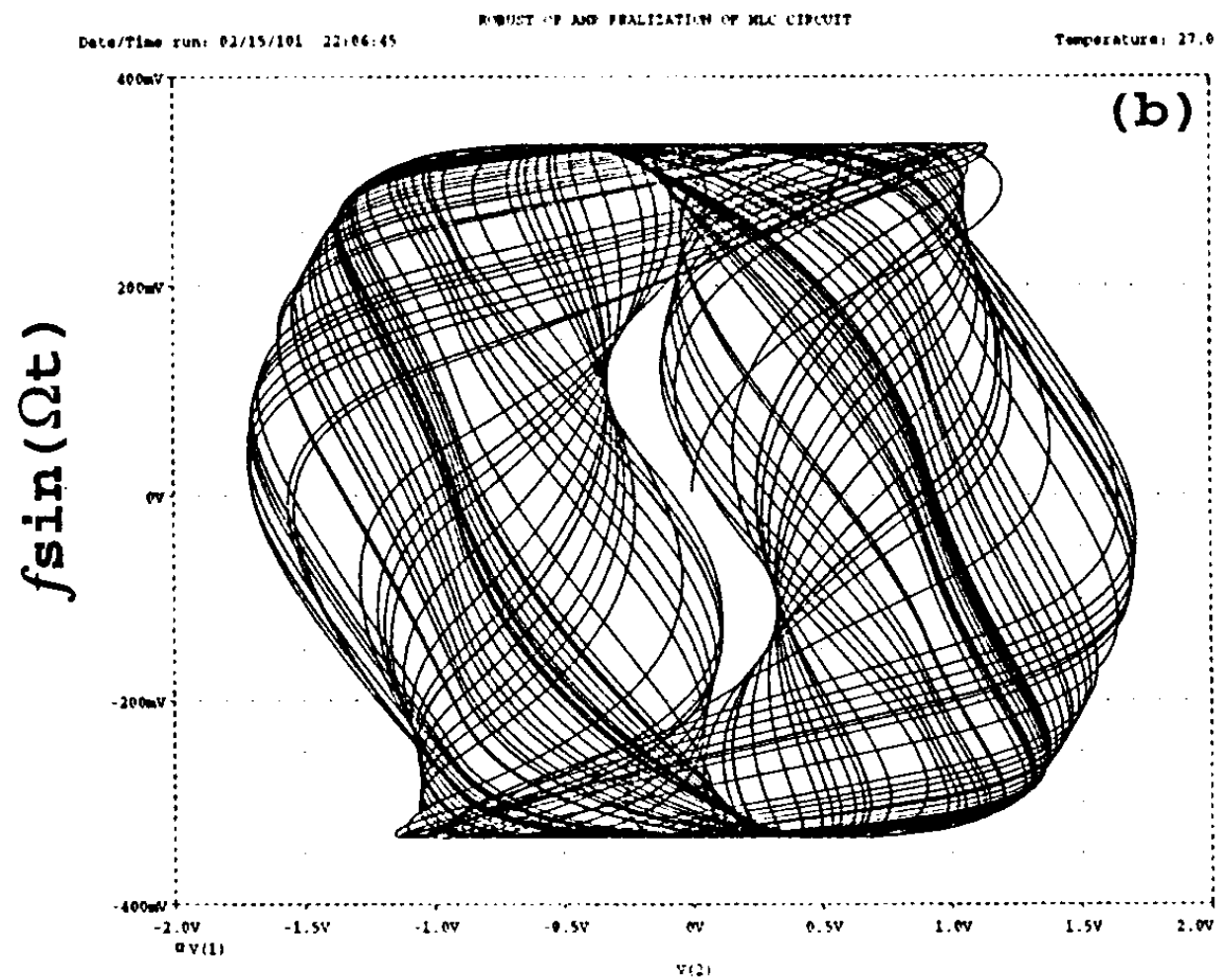

voltage

Fig. 22. (Continued)

\section{Analytical Studies of the MLC Variant Circuit: Linear Stability of Fixed Points, Bifurcations and Exact Solutions}

In this section we will briefly investigate the analytical aspects of the MLC variant circuit equation (4) along the lines of the MLC circuit equation (2) [Lakshmanan \& Murali, 1996]. To start with we note that in the autonomous case $(F=0)$, Eq. (4) is symmetric with respect to the origin so that it is invariant under the transformation

$$
(x, y) \rightarrow(X, Y)=(-x,-y)
$$

In this case, the equilibrium points can be obtained from the conditions,

$$
\begin{array}{r}
-x-y-g(x)=0, \\
\beta x=0 .
\end{array}
$$

It follows from the form of $g(x)$, Eq. (4c), that (4) has a unique equilibrium in each of the following three regions

$$
\begin{array}{cl}
D_{1} & =\{(x, y) \mid \quad x \geq 1\}, \\
D_{0} & =\{(x, y)|\quad| x \mid \leq 1\}, \\
D_{-1} & =\{(x, y) \mid \quad x \leq-1\} .
\end{array}
$$

The equilibria $\left(x_{0}, y_{0}\right)$ are explicitly given by

$$
\begin{aligned}
\text { (i) } & P^{+}=(0, b-a) \in D_{1}, \\
\text { (ii) } \quad O & =(0,0) \in D_{0}, \\
\text { (iii) } & P^{-}=(0, a-b) \in D_{-1} .
\end{aligned}
$$

In each of the regions $D_{1}, D_{0}$ and $D_{-1}$, Eq. (4) is linear when $F=0$. In fact, letting

$$
X=\left(x_{0}, y_{0}\right)
$$

for the region $D_{0}$ the stability determining eigenvalues are calculated from the stability matrix

$$
A_{0}=A(\beta, a)=\left(\begin{array}{cr}
-1-a & -1 \\
\beta & 0
\end{array}\right)
$$


as (with $a=-1.127, \beta=0.05)$

$$
\begin{aligned}
& \lambda_{1}=0.0635+i(0.21440), \\
& \lambda_{2}=0.0635-i(0.21440) .
\end{aligned}
$$

This indicates that $\left(x_{0}, y_{0}\right)=O \in D_{0}$ is an unstable spiral fixed point.

Similarly, from the stability matrix

$$
A_{ \pm}=A(\beta, b)=\left(\begin{array}{cr}
-1-b & -1 \\
\beta & 0
\end{array}\right)
$$

(with $b=-0.6047, \beta=0.05$ ) associated with the regions $D_{1}$ and $D_{-1}$, one can check that the system has a pair of complex-conjugate eigenvalues $\left(\lambda_{1}, \lambda_{2}=\lambda_{1}^{*}\right)$ with negative real parts: $\lambda_{1}=$ $-0.197625+i(0.1046)$ and $\lambda_{2}=-0.197625-$ $i(0.1046)$. So both $P^{+}$and $P^{-}$are stable spiral fixed points.

Thus one finds that as long as the initial conditions are confined to the region $D_{0}$, due to the unstable spiral nature of the fixed point $\mathrm{O}$, a stable limit cycle results. However, if the initial conditions are chosen in the regions $D_{ \pm}$, the system will end up in one of the fixed points $P^{+}$or $P^{-}$as the case may be, since they correspond to stable spirals. When the forcing periodic signal is included $(F>0)$ in the variant MLC circuit equation (4), there is an interaction between the limit cycle motion of the system and the external periodic signal, resulting in a Hopf bifurcation which gives rise to a quasiperiodic solution as observed in the experimental and numerical studies. It may be noted that in contrast to the above that in the case of the MLC circuit equation (2), for the chosen parameter values, the fixed point $\mathrm{O}$ is a hyperbolic fixed point, while $P^{+}$and $P^{-}$are stable spiral fixed points. So no limit cycle motion occurs in the MLC circuit case (see [Murali \& Lakshmanan, 1996]).

Actually, Eq. (4) can be explicitly integrated in terms of elementary functions in each of the three regions $D_{0}, D_{1}$ and $D_{-1}$ and matched across the boundaries to obtain the full solution. The details are as follows.

Let $\left[x\left(t ; t_{0}, x_{0}, y_{0}\right), y\left(t ; t_{0}, x_{0}, y_{0}\right)\right]$ be the solution to Eq. (4) of which the initial condition is given by $(t, x, y)=\left(t_{0}, x_{0}, y_{0}\right)$. Since Eq. (4) is piecewise-linear, the solution in each of the regions can be obtained explicitly as follows:

(i) Region $D_{0}(|x| \leq 1)$ :

Here, $g(x)=a x$ and Eq. (4) or (5) becomes

$$
\ddot{y}+(1+a) \dot{y}+\beta y=\beta F \sin (\omega t),
$$

The general solution to (14) is

$$
\begin{aligned}
y(t)= & e^{u t}[A \cos v t+B \sin v t]+C \sin \omega t \\
& +D \cos \omega t,
\end{aligned}
$$

where $A$ and $B$ are integration constants and

$$
\begin{aligned}
u & =\frac{\left(-k+\sqrt{k^{2}-4 \beta}\right)}{2}, \\
v & =\frac{\left(-k-\sqrt{k^{2}-4 \beta}\right)}{2}, \\
C & =\frac{\left(\beta F\left(\beta-\omega^{2}\right)\right)}{\left(k^{2} \omega^{2}+\left(\beta-\omega^{2}\right)^{2}\right)}, \\
D & =\frac{-\beta F \omega k}{\left(k^{2} \omega^{2}+\left(\beta-\omega^{2}\right)^{2}\right)}, \\
k & =1+a .
\end{aligned}
$$

Then, $x(t)$ is obtained from (4) as

$$
\begin{aligned}
x(t)= & \frac{1}{\beta}\left\{u e^{u t}(A \cos v t+B \sin v t)\right. \\
& +e^{u t}(B v \cos v t-A v \sin v t)+C \omega \cos \omega t \\
& -D \omega \sin \omega t\} .
\end{aligned}
$$

The constants $A$ and $B$ in (15) can be fixed from the initial condition $\left(x_{0}, y_{0}\right)$, provided it is in $D_{0}$.

(ii) Region $D_{1}(|x| \geq 1)$ :

Here, $g(x)=b x+a-b$. Then Eq. (5) becomes

$$
\ddot{y}+(1+b) \dot{y}+\beta y=\beta(b-a)+\beta F \sin (\omega t),
$$

The general solution to (18) is

$$
\begin{aligned}
y(t)= & e^{u t}\left[A_{1} \cos v t+B_{1} \sin v t\right]+C \sin \omega t \\
& +D \cos \omega t+(b-a),
\end{aligned}
$$

and $x(t)$ is obtained from Eq. (4). In Eq. (19), $A_{1}$ and $B_{1}$ are arbitrary constants while $u, v, C$ and $D$ are as defined in Eq. (16), except now we have the parameter $k$ defined as $k=1+b$.

(iii) Region $D_{-1}(x \leq-1)$ :

Here, $g(x)=b x-a+b$. Then Eq. (4) can be solved again by carrying out a similar analysis as above, and the general solution $y(t)$ becomes

$$
\begin{aligned}
y(t)= & e^{u t}\left[A_{2} \cos v t+B_{2} \sin v t\right]+C \sin \omega t \\
& +D \cos \omega t+(a-b),
\end{aligned}
$$


where again $A_{2}$ and $B_{2}$ are arbitrary constants and $x(t)$ is obtained from Eq. (4) as before.

Thus, for example if we start with the initial condition in $D_{0}$, the arbitrary constants $A$ and $B$ in Eq. (15) get fixed. Then $x(t)$ evolves as given by Eq. (17) up to either $t=T_{1}$, when $x\left(T_{1}\right)=1$ and $\dot{x}\left(T_{1}\right)>0$ or $t=T_{1}^{\prime}$ when $x\left(T_{1}^{\prime}\right)=-1$ and $\dot{x}\left(T_{1}^{\prime}\right)<0$. The value of $T_{1}$ and $T_{1}^{\prime}$ are obtained numerically. Knowing whether $T_{1}>T_{1}^{\prime}$ or $T_{1}<T_{1}^{\prime}$ we can determine the next region of interest $\left(D_{1}\right.$ or $D_{-1}$ ) and the arbitrary constants of the solutions of that region can be fixed by matching the solutions across the boundary. This procedure can be continued for each successive crossing. In this way, explicit solutions can be obtained in each of the regions $D_{0}, D_{1}$ and $D_{-1}$. A similar procedure can be followed if the initial condition is either in $D_{1}$ or $D_{-1}$ by making use of the solution (19) or (20), respectively. However, it is clear that sensitive dependence on initial conditions is introduced in each of these crossings at appropriate parameter regimes during the inversion procedure of finding $T_{1}, T_{1}^{\prime}, T_{2}, T_{2}^{\prime}, \ldots$, etc. from the solutions. We have verified that all the numerical results reported earlier in the paper for various parametric regimes also follow from the explicit solutions reported in this section.

\section{Conclusions}

In this paper, we have shown by a detailed experimental, numerical and analytical investigation that the familiar parallel LCR circuit to which the Chua's diode is connected parallely can exhibit a surprisingly wide range of bifurcation and chaos phenomena associated with several nonlinear dynamical systems. Particularly it exhibits quasiperiodic attractors, torus breakdown route to chaos, period-adding and Farey sequences, antimonotonicity and bubbling, intermittency route to chaos and so on, besides the familiar period-doubling route. The underlying dynamical phenomena covered seems to be much wider than the standard MLC circuit, where the series LCR circuit and the Chua's diode are connected in parallel.

The existence of such simple nonautonomous nonlinear electronic circuits exhibiting rich varieties of bifurcation phenomena can be profitably used in areas where nonlinear electronic circuits are used in applications such as controlling, synchronization, secure communication, cryptography, and so on.
Further, the study of the dynamics of arrays of such circuits will be very valuable to understand the spatiotemporal patterns arising from the interaction of such diverse dynamical states. Work is in progress along these lines.

\section{Acknowledgments}

A substantial portion of this work has been done in collaboration with K. Murali, whose suggestions and advice are gratefully acknowledged. A. Venkatesan and P. Muruganandam have helped very much at various stages of the work. This work forms part of a research project supported by the Department of Science and Technology, Government of India.

\section{References}

Bier, M. \& Bountis, T. C. [1984] "Remerging Feigenbaum trees in dynamical systems," Phys. Lett. A104, 239-244.

Chua, L. O., Yao, Y. \& Yang, Q. [1986] "Devil's staircase route to chaos in a nonlinear circuit," Int. J. Circuit Th. Appl. 14, 315-329.

Chua, L. O., Desoer, C. A. \& Kuh, E. S. [1987] Linear and Nonlinear Circuits (McGraw-Hill, NY).

Cruz, J. M. \& Chua, L. O. [1992] "A CMOS IC nonlinear resistor for Chua's circuit," IEEE Trans. Circuits Syst. I 39, 985-995.

Inaba, N. \& Mori, S. [1991] "Chaos via torus break down in a piecewise-linear forced van der Pol oscillator with a diode," IEEE Trans. Circuits Syst. I 38, 398-409.

Inaba, N. \& Mori, S. [1992] "Folded torus in the forced Rayleigh oscillator with a diode pair," IEEE Trans. Circuits Syst. I 39, 402-411.

Kaneko, K. [1986] Collapse of Tori and Genesis of Chaos in Dissipative Systems (World Scientific, Singapore).

Kennedy, M. P., Kreig, K. P. \& Chua, L. O [1989] "The devil's staircase: The electrical engineer's fractal," IEEE Trans. Circuits Syst. 36, 1133-1139.

Kennedy, M. P. [1992] "Robust op amp realization of Chua's circuit," Frequenz 46, 66-80.

Kypriandis, I. M., Stouboulos, I. N., Haralabids, P. \& Bountis, T. [2000] "Antimonotonicity and chaotic dynamics in a fourth order autonomous nonlinear electrical circuit," Int. J. Bifurcation and Chaos 10(8), 1903-1915.

Lakshmanan, M. \& Murali, K. [1996] Chaos in Nonlinear Oscillators: Controlling and Synchronization (World Scientific, Singapore).

Madan, R. [1993] "Special issue on Chua's circuit: A paradigm for chaos, Part I \& II," J. Circuit Syst. Comput. 3. 
Manneville, P. \& Pomeau, Y. [1980] "Different ways to turbulence in dissipative systems," Physica D1, 219-226.

Matsumoto, T., Chua, L. O. \& Tokunaga, R. [1987] "Chaos via torus breakdown," IEEE Trans. Circuits Syst. CAS34, 240-253.

Murali, K. \& Lakshmanan, M. [1991] "Bifurcation and chaos of the sinusoidally driven Chua's circuit," Int. J. Bifurcation and Chaos 1, 369-384.

Murali, K. \& Lakshmanan, M. [1992] "Transition from quasiperiodicity to chaos and devil's staircase structures of the driven Chua's circuit," Int. J. Bifurcation and Chaos 2, 621-632.

Murali, K., Lakshmanan, M. \& Chua, L. O. [1994a] "The simplest dissipative nonautonomous chaotic circuit," IEEE Trans. Circuits Syst. I 41, 462-463.

Murali, K., Lakshmanan, M. \& Chua, L. O. [1994b] "Bifurcation and chaos in the simplest dissipative nonautonomous chaotic circuit," Int. J. Bifurcation and Chaos 4, 1511-1524.

Ott, E. [1993] Chaos in Dynamical Systems (Cambridge University Press, London).

Roberts, W. \& Sedra, S. [1997] SPICE (Oxford University Press, NY).

Schuster, H. G. [1988] Deterministic Chaos: An Introduction (Physik-Verlag, Weinheim).

Thamilmaran, K., Lakshmanan, M. \& Murali, K. [2000] "Rich variety of bifurcations and chaos in a variant of Murali-Lakshmnan-Chua circuit," Int. J. Bifurcation and Chaos 10(7), 1175-1180.

Thompson, J. M. T. \& Stewart, H. B. [1988] Nonlinear Dynamics and Chaos (John Wiley, Chichester).

Venkatesan, A. \& Lakshmanan, M. [1997] "Bifurcation and chaos in the double-well Duffing-van der Pol oscillator: Numerical and analytical studies," Phys. Rev. E56, 6321-6330. 